\title{
Blood flow simulations in the pulmonary bifurcation in relation to adult patients with repaired tetralogy of Fallot
}

\author{
Maria Boumpouli ${ }^{1}$, Mark H.D. Danton ${ }^{1,2}$, Terence Gourlay ${ }^{1}$ and Asimina Kazakidi ${ }^{1, *}$ \\ ${ }^{1}$ Department of Biomedical Engineering, University of Strathclyde, Glasgow G4 0NW, UK \\ ${ }^{2}$ Scottish Adult Congenital Cardiac Service, Golden Jubilee National Hospital, Clydebank G81 4DY, UK
}

\begin{abstract}
Understanding the haemodynamic environment of the pulmonary bifurcation is important in adults with repaired conotruncal congenital heart disease. In these patients, dysfunction of the pulmonary valve and narrowing of the branch pulmonary arteries are common and can have serious clinical consequences. The aim of this study was to numerically investigate the underlying blood flow characteristics in the pulmonary trunk under a range of simplified conditions. For that, an in-depth analysis was conducted in idealised two-dimensional geometries that facilitate parametric investigation of healthy and abnormal conditions. Subtle variations in morphology influenced the haemodynamic environment and wall shear stress distribution. The pressure in the left pulmonary artery was generally higher than that in the right and main arteries, but was markedly reduced in the presence of a local stenosis. Different downstream pressure conditions altered the branch flow ratio, from 50:50\% to more realistic 60:40\% ratios in the right and left pulmonary artery, respectively. Despite some simplifications, this study highlights some previously undocumented aspects of the flow in bifurcating geometries, by clarifying the role of the stagnation point location on wall shear stress and differential branch pressures. In addition, measurements of the mean pressure ratios in the pulmonary bifurcation are discussed in the context of a new haemodynamic index which could potentially contribute to the assessment of left pulmonary artery stenosis in tetralogy of Fallot patients. Further studies are required to confirm the results in patient-specific models with personalised physiological flow conditions.
\end{abstract}

Keywords: Heamodynamics; Computational fluid dynamics; Numerical simulations; Congenital heart disease; Tetralogy of Fallot

\section{List of abbreviations}

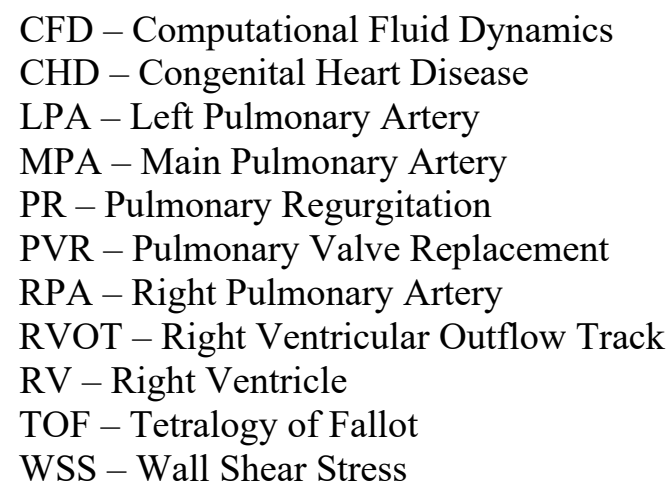

\section{Introduction}

Congenital Heart Disease (CHD) lesions occur during embryonic development and result in structural abnormalities of the heart [1]. In particular, tetralogy of Fallot (TOF), one of the common cyanotic lesions, comprises four morphological cardiac defects: a ventricular septal defect, overriding aorta, pulmonary stenosis, and right ventricular hypertrophy [2]. Surgical correction performed in infancy provides a bi-ventricular circulation and normal arterial saturations. With advancements in medical paediatric healthcare, the expected survival and quality of life have improved immensely, similar to normal population. However, as these patients progress into adulthood, they are at risk of late complications and further re-interventions $[3,4]$. 
Pulmonary regurgitation (PR) and branch pulmonary obstruction are the most common indications for reintervention, including pulmonary valve replacement (PVR) and arterial stenting [3, 4]. PR involves the diastolic reversal of blood flow into the right ventricle (RV) as a consequence of loss of pulmonary valve competence. In TOF patients, PR occurs due to long-term postoperative outcome of repaired right ventricular outflow tract (RVOT) obstruction during infancy, and the necessary surgical opening of the dysplastic valve. Although PR can be tolerated for an extended period of time, if mild or moderate, it typically results in right ventricular dilatation and dysfunction, exercise intolerance, and ventricular and atrial arrhythmias, when prolonged and severe $[5,6]$. Branch pulmonary obstruction is most commonly observed in the left pulmonary artery (LPA) [5] in the form of a local stenosis. Proximal LPA obstruction can also occur with general artery hypoplasia in pulmonary atresia and following complex repair of nonconfluent arteries, that is, when the right and the left branches are not interconnected and originate separately [7], or artery kinking, when pulmonary regurgitation is present in patients with dilated RVOT. The dilatation of the right ventricle and the pulmonary trunk, due to PR, restrict the RVOT, which turns cranially and left-laterally, causing the angle between the pulmonary trunk and LPA to become acute and eventually form LPA kinking. As a consequence of such morphological differentiations, RV afterload increases, which may intensify PR, resulting in turn in further enlargement of the right ventricle and kinking. It is, hence, likely that a twoway link between pulmonary regurgitation and LPA kinking may be present $[8,9]$.

Pulmonary valve replacement (PVR) is an operation performed to prevent ongoing volume overloading in the right ventricle and dilatation. It is deemed necessary when PR and LPA kinking are present to preserve the right ventricular function. PVR is recommended prior to the onset of significant clinical symptoms and right ventricular dysfunction, to increase the likelihood of successful RV remodelling. The assessment of the optimal timing for PVR is, therefore, crucial and remains a key challenge to clinicians, relying their decision upon balancing the risk between RV dysfunction and the finite lifespan of the available bioprosthetic valves used in PVR $[4,6]$. There is yet no consensus on the reliability and effectiveness of current indices in the clinical practice $[6,10]$, however recent studies have observed that PR is associated with differential retrograde flow of various flow splits in the left and right branch pulmonary arteries in patients with repaired TOF, irrespective of the presence of stenosis $[5,11]$. To clarify this, a better understanding of the subtle haemodynamic alterations in the pulmonary arterial bifurcation is required, with the scope to contribute towards a more accurate evaluation of the right timing for PVR and the prognosis and treatment of the above conditions.

Optimisation of flow delivered to the daughter branches of an arterial junction is essential in order to ensure that blood vessels supply the peripheral tissue and organs with sufficient amount of blood, compared to the energy consumed by the tissue and organs [12]. Murray's law, an early mathematical model, makes use of the branches radii in order to optimise work, through minimisation of energy consumption at bifurcations [13]. However, there are many fluid dynamic principles that can affect fluid flow and need to be taken into account. Several previous studies on physiological flows in branching arteries $[12,14]$ have demonstrated flow separation near branch entrances and the formation of recirculating zones, with fluid particles trapped within, due to their inability to re-enter the mainstream flow $[12,15]$. Nevertheless, the haemodynamics of arterial bifurcations may further be affected by local morphology, such as the branching angle, and various other factors, including nonNewtonian rheology, turbulent flow, time-dependency, and loss of wall deformability [15]. The pulmonary bifurcation in repaired TOF patients is a particular junction with varied and often abnormal geometrical and boundary conditions, and their detailed effects on blood flow need further analysis.

Computational fluid dynamic (CFD) methods are valuable tools for non-invasively simulating blood flow through vessels and allow for the examination of various effects that may influence flow development. Computational models are increasingly used to support clinical diagnosis, treatment, and surgical planning, through the quantification of hemodynamic parameters, such as velocity, pressure, and wall shear stress, and the investigation of blood flow patterns. Previous studies on blood flow in the pulmonary arteries have shown that in healthy subjects the pulmonary bifurcation is hemodynamically efficient with small separation of flow along the inner wall [16]. However, in congenital heart patients, reversal of flow has been observed in the LPA which precedes and contributes to pulmonary valve regurgitation [17], while flow becomes more disturbed for decreasing branching 
angle [3, 17-20]. In most of these studies, blood was assumed as a Newtonian fluid, which is an accepted approximation in large arteries [17].

In the present work, computational fluid dynamics tools were used to thoroughly investigate blood flow in idealised two-dimensional models of the pulmonary bifurcation. Although simplified, the use of such geometries allow for a detailed parametric analysis of the influence of various conditions on blood flow, improving our understanding of the underlying flow mechanisms of more complex systems, while being much less computationally demanding [21]. Different geometries were considered here, representative of healthy and abnormal morphologies of the pulmonary trunk. The novelty of this work lies in the extended analysis of bifurcating flows in relation to TOF patients and a hemodynamic assessment in the pulmonary arteries, which may be useful to clinicians and adult congenital heart patients.

The remainder of this paper is organised as follows: Section 2 describes the numerical methodology, including model generation and boundary conditions; Section 3 presents the main results of this study, divided in the effects of geometry and outflow boundary conditions; Section 4 discusses the results in the context of previous studies, and Section 5 concludes this work with some final remarks.

\section{Computational approximations}

\subsection{Models of the pulmonary bifurcation}

Nine different geometries of the pulmonary bifurcation were created in the two-dimensional space in order to investigate the effects of branching angle, branch origin, and LPA stenosis (Fig. 1). These simplified models make it possible to analyse healthy and abnormal flow conditions and identify critical parameters. The diameter of the main pulmonary artery (MPA), denoted as $D$, was assumed 2.6 $\mathrm{cm}$, while both daughter branch diameters were taken, for the majority of the models, equal to $2 \mathrm{~cm}$ (denoted as $d$ ). These values are within the normal physiological ranges, without attempting to replicate exact patient cases [22]. The MPA length was assumed $\sim 7.7 D$, while the LPA and RPA lengths were chosen to be $\sim 115 D$, the minimum branch length at which no effect on the maximum wall shear stress (WSS) from the boundaries was found (Fig. 1 shows only a section of each model). The nine geometries are as follows (Table 1):

a. A T-Junction where the angle between the MPA and daughter branches is $90^{\circ}$ (denoted as $\vartheta_{1}$ for RPA and $\vartheta_{2}$ for LPA); this geometry represents the shape of the pulmonary bifurcation in patients repaired for both LPA and RPA stenosis [23], or in the context of pulmonary atresia with RV pulmonary artery conduit and/or confluence constriction (Fig. $1 \mathrm{i}$ ).

b. An asymmetric Y-Junction with $\vartheta_{1}=100^{\circ}$ and $\vartheta_{2}=150^{\circ}$; this geometry can be seen as the normal shape for the pulmonary bifurcation, assuming a common origin of the two branches, at point $O$ (Fig. 1 ii). An axial black-blood MRI image of the pulmonary bifurcation of a healthy volunteer (male, 27 years old) can be seen in Fig. 2a obtained with ethical approval from the Glasgow CRIF approval group, and written informed consent, REC name WoS REC3, reference 16/WS/0220).

c. An asymmetric Y-Junction, with $\theta_{1}, \theta_{2}$ as in (ii), which assumes a displaced origin for the LPA centreline, by $0.5 \mathrm{~cm}(\sim 0.19 D)$ upstream of point $\mathrm{O}$ (Fig. 1 iii), and can also be considered physiological.

d. An asymmetric Y-Junction, with $\vartheta_{1}, \vartheta_{2}$ as in (ii) and displaced origins for both LPA and RPA, by $0.3 \mathrm{~cm}(\sim 0.12 D)$ downstream and $1 \mathrm{~cm}(\sim 0.39 D)$ upstream of point $\mathrm{O}$, respectively (Fig. 1 iv).

e. An asymmetric Y-Junction, with $\vartheta_{1}, \vartheta_{2}$ as in (ii), but with a displaced origin and a reduced diameter for LPA by $1 \mathrm{~cm}(0.5 d)$, representing a uniform small calibre of the pulmonary branch or, in the context of this work, a hypoplastic branch (Fig. $1 \mathrm{v}$ ) [24].

f. A symmetric Y-Junction with $\vartheta_{1}=\vartheta_{2}=120^{\circ}$, representing the pulmonary bifurcation of repaired patients with TOF, including cases where the MPA may be directly attached to the RV without conduit (Fig. 1 vi) [23].

g. A symmetric Y-Junction, with $\vartheta_{1}=\vartheta_{2}$ as in (vi), and a local $50 \%$ asymmetric stenosis within the LPA, positioned $1.7 \mathrm{~cm}(\sim 0.65 D)$ downstream of the pulmonary bifurcation. This geometry represents the case of LPA kinking (Fig. 1 vii) [23]. 
h. A symmetric Y-Junction, with $\vartheta_{1}=\vartheta_{2}$ as in (vi), and a local $50 \%$ symmetric stenosis within the LPA, positioned $1.7 \mathrm{~cm}(\sim 0.65 D)$ downstream of the pulmonary bifurcation (Fig. 1 viii).

i. A symmetric Y-Junction, with $\vartheta_{1}=\vartheta_{2}$ as in (v), but with a displaced origin and a hypoplastic LPA [24], with a reduced diameter by $1 \mathrm{~cm}(0.5 d)$ (Fig. 1 ix).

Table 1: Characteristics of the nine different models created to represent the pulmonary bifurcation.

\begin{tabular}{|c|c|c|c|c|c|c|}
\hline \multirow{2}{*}{ Model } & \multirow{2}{*}{ Type } & \multicolumn{2}{|c|}{ Branching Angle } & \multirow{2}{*}{$\begin{array}{c}\text { Branch Origin in } \\
\text { relation to point } \\
0\end{array}$} & \multirow{2}{*}{ LPA } & \multirow{2}{*}{$\begin{array}{c}\text { Clinical } \\
\text { Relevance }\end{array}$} \\
\hline & & $\vartheta_{1}$ & $\vartheta_{2}$ & & & \\
\hline (i) & T-Junction & $90^{\circ}$ & $90^{\circ}$ & Common & - & $\begin{array}{l}\text { Repaired } \\
\text { TOF }\end{array}$ \\
\hline (ii) & \multirow{4}{*}{$\begin{array}{l}\text { Asymmetric } \\
\text { Y-Junction }\end{array}$} & \multirow{4}{*}{$100^{\circ}$} & \multirow{4}{*}{$150^{\circ}$} & Common & - & \multirow{3}{*}{$\begin{array}{l}\text { Physiological } \\
\text { case }\end{array}$} \\
\hline (iii) & & & & Displaced for LPA & - & \\
\hline (iv) & & & & $\begin{array}{l}\text { Displaced for both } \\
\text { branches }\end{array}$ & - & \\
\hline (v) & & & & Displaced for LPA & Hypoplastic & $\begin{array}{l}\text { Hypoplastic } \\
\text { LPA }\end{array}$ \\
\hline (vi) & \multirow{4}{*}{$\begin{array}{l}\text { Symmetric Y- } \\
\text { Junction }\end{array}$} & \multirow{4}{*}{$120^{\circ}$} & \multirow{4}{*}{$120^{\circ}$} & \multirow{3}{*}{ Common } & - & $\begin{array}{l}\text { Repaired } \\
\text { TOF }\end{array}$ \\
\hline (vii) & & & & & $\begin{array}{c}\text { Local } \\
\text { asymmetric } \\
\text { stenosis }\end{array}$ & $\begin{array}{l}\text { LPA kinking } \\
\text { - asymmetric } \\
\text { stenosis }\end{array}$ \\
\hline (viii) & & & & & $\begin{array}{l}\text { Local } \\
\text { symmetric } \\
\text { stenosis }\end{array}$ & $\begin{array}{c}\text { Branch LPA } \\
\text { stenosis - } \\
\text { symmetric } \\
\text { stenosis }\end{array}$ \\
\hline (ix) & & & & Displaced for LPA & Hypoplastic & $\begin{array}{l}\text { Hypoplastic } \\
\text { LPA }\end{array}$ \\
\hline
\end{tabular}



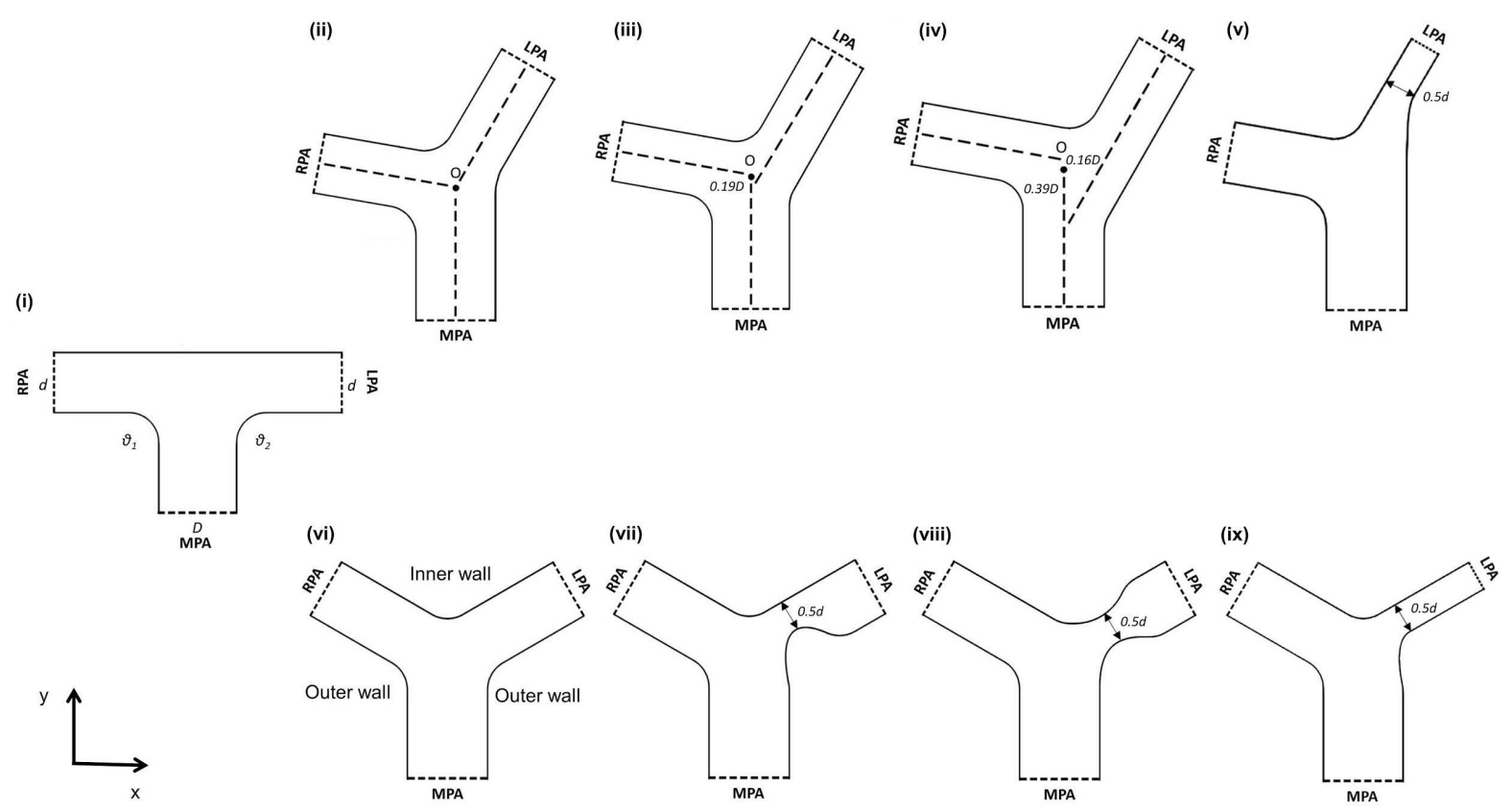

Fig. 1: Schematic representation of nine different models of the pulmonary bifurcation. (i) T-Junction; (ii) asymmetric YJunction with common branch origin at point O; (iii)-(iv) asymmetric Y-Junctions with displaced branch origins; (v) asymmetric Y-Junction with displaced origin and hypoplastic LPA; (vi) symmetric Y-Junction; (vii)-(ix) symmetric Y-Junctions with local stenosis and hypoplastic LPA, respectively. The inner and outer walls are annotated on (vi).

\subsection{Numerical approximations}

The computational meshes for all geometries of Fig. 1 were generated using the commercial software ANSA v17.1 (BETA CAE Systems). Each mesh consisted primarily of quadrilateral elements, with a few triangular elements in the middle of the bifurcation (Fig. 2b). A boundary layer mesh was also added near the arterial wall boundaries to capture the viscous effects, which included 17 layers with the first layer being at a distance of $\sim 0.002 D$ away from the wall. This was determined based on a low $\mathrm{y}+$ wall treatment, calculated using the formula $\rho \sqrt{\tau_{w} / \rho} * y / \mu$, where $\rho$ is the fluid density (1060 $\left.\mathrm{kg} / \mathrm{m}^{3}\right), \tau_{w}$ is the wall shear stress, $y$ is the height of the first near-wall cell, and $\mu$ is the dynamic viscosity $\left(4 * 10^{-3-} \mathrm{Pas}\right.$ ) [25], and was equal to 0.18 . This implies that the viscous boundary layer is sufficiently resolved within the fine layered mesh. The total number of elements for the nine geometries of Fig. 1 was between $90000-125000$. According to a grid independence analysis performed previously by the authors [26], this number of elements was deemed sufficient for the flow regimes examined in this work. In addition, the use of triangular or primarily quadrilateral elements in the mesh was compared and showed no difference in the integral of velocity at the entrance of the bifurcation (as shown in [26]). The latter type of elements was chosen to avoid non-orthogonality issues.

For the simulations presented here, blood flow was considered Newtonian, laminar and incompressible, governed by the Navier-Stokes equations:

$$
\begin{aligned}
\nabla \cdot u & =0 \\
\rho \frac{\partial u}{\partial t}+\rho(u \cdot \nabla) u & =-\nabla p+\mu \nabla^{2} u
\end{aligned}
$$

where $u$ is the velocity vector and $p$ is the static pressure [26]. In the models of Fig. 1, a steady, parabolic inlet velocity profile was assigned at the MPA $[15,27]$, according to the equation:

$$
u_{\text {par }}(x)=\frac{3}{2} U\left(1-\frac{4 x^{2}}{D^{2}}\right)
$$

with mean velocity $U=0.1 \mathrm{~m} / \mathrm{s}$, an average physiological value noted in healthy subjects [28, 29], and rigid walls. The Reynolds number $\left(\operatorname{Re}_{D}=U D / v\right)$, calculated based on the MPA diameter, was 650 for all cases. The semi-implicit method for pressure linked equations (SIMPLE) algorithm, adopted by many CFD studies [30], of the OpenFOAM ${ }^{\circledR}$ open-source library was utilised for the pressure-velocity coupling in all the numerical calculations. The Gauss linear gradient and the second order bounded 
Gauss linear upwind divergence numerical schemes were used. Residual control was set to $10^{-6}$ for pressure and velocity.

To account for the unsteady nature of blood flow, a sinusoidal variation of the velocity profile at the MPA inlet was assumed for the geometry of Fig. 1 ix, based on the following expression:

$$
u(x, t)=u_{\text {par }}[1+\sin (2 \pi f t)]
$$

where $f$ is the frequency, assumed as $2 \mathrm{~Hz}$ [31]. Time-averaged results over a single cycle were compared with the steady-state numerical results for the same mean velocity. A combination of the SIMPLE and the pressure implicit with splitting of operators (PISO) algorithms for pressure-velocity coupling within OpenFOAM ${ }^{\circledR}$ (PIMPLE) was utilised for the unsteady numerical calculations.
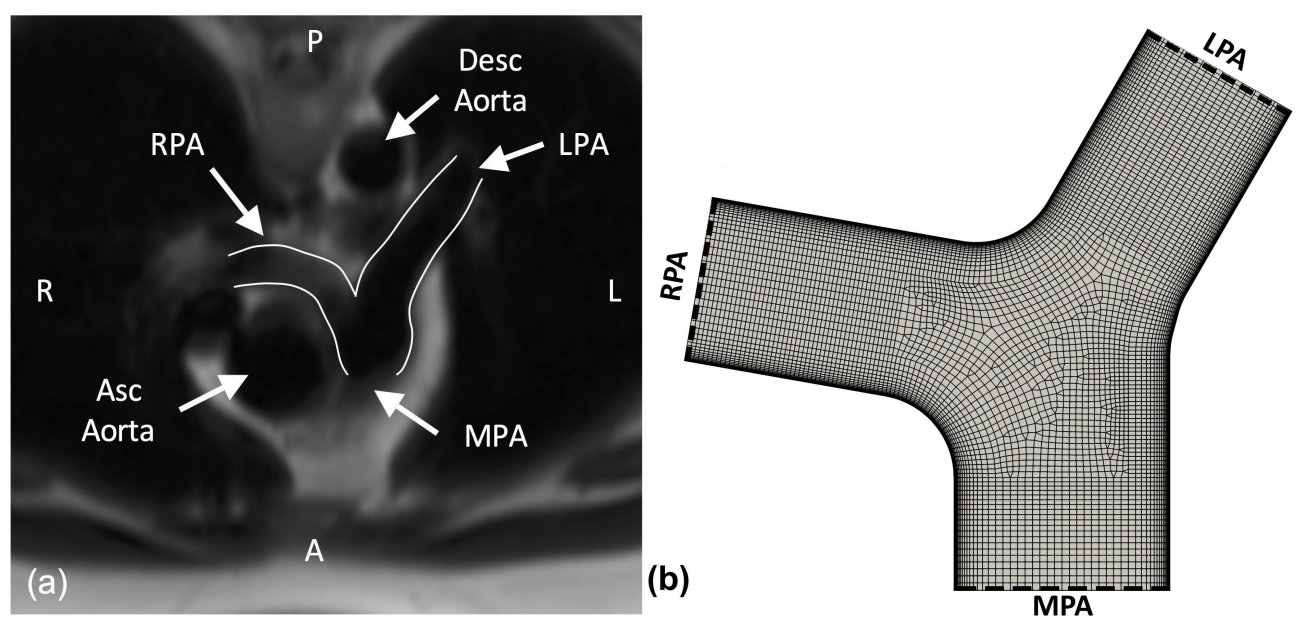

Fig. 2: (a) An axial black-blood MRI image from a healthy male volunteer (27 years old), overlaid with an outline of the inner and outer walls of the pulmonary bifurcation. (b) Computational mesh for the geometry of Fig. 1 ii, consisting mainly of quadrilateral elements, a few triangular elements in the middle of the bifurcation and a boundary layer near the wall.

\subsection{Effect of outlet boundary conditions}

For the geometries of Fig. 1, a condition of zero-pressure was initially applied at the LPA and RPA outlets [3], which corresponds to a mean pressure at rest of 8-20 mmHg. The zero-pressure assumption is commonly used in simplified geometries without available patient-specific information of flow and pressure waveforms or peripheral resistances [32]. However, in order to acquire more clinically relevant results and flow splits in the daughter branches, an investigation on the outflow boundary condition was conducted, in three different ways:

Prescribed pressure outlet conditions: Using the symmetric Y-Junction geometry (Fig. 1 vi), first, the RPA branch was elongated to double its original length, since physiologically the RPA is longer than the LPA [33], with zero pressure assigned at both outlets; second, a pressure difference of $|\triangle P|=\left|P_{R P A}-P_{L P A}\right|$, normalised by $0.5 \rho U^{2}$, was prescribed at the branch outlets. A range of different $|\triangle P|$ values were tested and the approximate values of 0.026 and 0.015 were chosen, since these pressure difference values provided more clinically relevant flow splits in the daughter branches, closer to $40: 60 \%[34]$.

Prescribed flow splits: Murray's Law formula can be used to calculate the flow expected in each branch outlet, based on the branch diameter, and, for the LPA, it could be expressed as:

$$
Q_{L P A} /\left(Q_{L P A}+Q_{R P A}\right)=\left[D_{L P A} /\left(D_{L P A}+D_{R P A}\right)\right]^{n}
$$

where $\mathrm{Q}_{\mathrm{LPA}}, \mathrm{Q}_{\mathrm{RPA}}$ are, respectively, the flow rates at the LPA and RPA branches, $\mathrm{D}_{\mathrm{LPA}}, \mathrm{D}_{\mathrm{RPA}}$ the branch diameters, while, customarily, $\mathrm{n}$ is considered equal to 3 .

Eq. 5 can be rewritten based on the "outflow splitting" method [35], which calculates the flow division locally for each bifurcation generation, assuming $n=2$. For the pulmonary bifurcation, this is expressed as: 


$$
Q_{L P A} / Q_{R P A}=\left(D_{L P A} / D_{R P A}\right)^{n}
$$

The symmetric Y-Junction model with the hypoplastic LPA (Fig. 1 ix) was used as a reference geometry for this investigation, due to the difference in the diameter of the daughter branches. Based on the conservation of mass, the flow splits were calculated as $\mathrm{Q}_{\mathrm{LPA}} / \mathrm{Q}_{\mathrm{RPA}}=(1 / 3)^{\mathrm{n}}$ with the Murray's Law, giving 11:89\%, and $\mathrm{Q}_{\mathrm{LPA}} / \mathrm{Q}_{\mathrm{RPA}}=(1 / 2)^{\mathrm{n}}$ with the "outflow splitting" method, resulting in 20:80\% flow split.

Lumped parameter models: A lumped model made of a peripheral resistance was coupled and implemented on each of the outlets of the symmetric hypoplastic model (Fig. 1 ix) to capture effects of the downstream vasculature [36]. The pressure, $p$, was calculated based on the flow rate, $Q$, and the resistance, $R$, according to the following equation:

$$
\mathrm{p}_{\mathrm{i}}=\mathrm{Q}_{\mathrm{i}} * \mathrm{R}_{\mathrm{i}}
$$

where index $i$ refers to the LPA and RPA branches, respectively. $\mathrm{R}_{\mathrm{LPA}}$ and $\mathrm{R}_{\mathrm{RPA}}$ were assumed both equal to 300 dyn s cm$~^{-5}$ [18], matching the distal resistance assumed in the three-element Windkessel model [16], described in the next paragraph. The effect of a pulsatile inlet flow condition was also investigated for the peripheral resistance boundary condition.

Furthermore, a three-element Windkessel (WK) lumped parameter model was coupled at the outlets of the geometries to represent the peripheral resistance and wall compliance of the pulmonary vessels [18], according to the following differential equation:

$$
p(t)+R_{d} C \frac{\partial P(t)}{\partial t}=\left(R_{p}+R_{d}\right) Q(t)+R_{p} R_{d} C * \frac{\partial Q(t)}{\partial t}
$$

where $R_{p}$ and $R_{d}$ are the proximal and distal resistances, and $\mathrm{C}$ is the capacitance, assumed as 40 dyn $\mathrm{s} \mathrm{cm}^{-5}, 300$ dyn s cm${ }^{-5}$, and $10^{-3} \mathrm{~cm}^{5} \mathrm{dyn}^{-1}$, respectively [18]. The geometry of Fig. 1 ix was used for this investigation, utilising the PIMPLE algorithm for pressure-velocity coupling within OpenFOAM ${ }^{\circledR}$, with the relaxation factors for pressure and velocity equal to 0.3 and 0.7 , respectively.

\subsection{Sensitivity analysis}

Further to the flow simulations in the geometries of Fig. 1, an additional sensitivity analysis was performed in the symmetric Y-Junction model (Fig. 1 vi) to investigate the effects of other boundary conditions on the original solution, including: the velocity profile at the inlet and the MPA length, time-dependent flow, use of turbulent flow model, effect of Reynolds number, a threedimensional model and non-Newtonian models. Details on the methodology and the results of this analysis can be found in the Supplementary Material.

\subsection{Post-processing}

To characterise the flow development in the arterial models, contours of velocity, velocity profiles and streamlines, WSS distribution along the inner wall of the bifurcation (connecting the LPA and RPA as shown in Fig. 1 vi), as well as pressure ratios and flow splits were analysed. Velocity values were non-dimensionalised by division with the mean MPA velocity $U$. WSS was non-dimensionalised with the value corresponding to the inlet WSS magnitude of a long straight segment that has the same diameter of MPA inlet and same inflow conditions as the pulmonary bifurcation model. For a twodimensional channel, this can be calculated for a Newtonian model with a fully developed axial velocity profile as $\tau_{w_{2 D}}=6 \mu U / D$ [37]. The calculation of flow splits was based on the velocity profiles at a distance of $3 \mathrm{~cm}(1.15 D)$ from the branch origin for each model. The time-averaged wall shear stress (TAWSS) was further used and was determined according to the following equation:

$$
\tau_{\text {mean }}=\frac{1}{T} \int_{0}^{T}\left|\tau_{w}\right| d t
$$

where $\left|\tau_{w}\right|$ is the magnitude of the instantaneous WSS vector. 


\subsection{Verification and validation}

Three different tests were employed to confirm the results of this study. First, a benchmark validation test was conducted based on a two-dimensional T-Junction model, as presented by Neofytou et al. (2014) [30], who compared their result with experimental measurements from the commonly used benchmark case of Liepsch and Moravec (1982) [38], with a mean velocity of $U=0.16 \mathrm{~m} / \mathrm{s}$, corresponding to a Reynolds number of 400. A good agreement was found between our computational results and those of [30], with a percentage difference in the velocity integrals below $0.2 \%$ for the parent channel and less than $1.4 \%$ for the daughter channels. Second, computational results obtained in the symmetric Y-Junction with OpenFOAM ${ }^{\circledR}$ appeared in very good agreement with calculations performed in ANSYS Fluent ${ }^{\circledR}$, for the same geometry and flow and boundary conditions (see Supplementary Material). More specifically, a percentage difference of $0.047 \%$ was quantified for the integral of velocity profiles positioned at the entrance of the bifurcation (at a distance $0.01 \mathrm{~m}$ from the origin $\mathrm{O}$ ). A comparison between the simpleFoam and the pisoFoam solvers of the OpenFOAM ${ }^{\circledR}$ library was conducted for the symmetric Y-Junction model, assuming steady flow. For a laminar flow model and a mean velocity of $U=0.1 \mathrm{~m} / \mathrm{s}$ at the MPA inlet, the length difference of the recirculation zone formed at the outer wall of the LPA was $1.3 \%$. For a turbulent flow model with a mean MPA velocity of $U$ $=0.5 \mathrm{~m} / \mathrm{s}$, the LPA recirculation length difference was $2 \%$ between the two solvers, based on the results from simpleFoam. The difference is slightly greater for the higher mean velocity and turbulent model, but still in good agreement, since the pisoFoam solver is more time-accurate for incompressible turbulent flows, using the PISO algorithm. Finally, a similar comparison was conducted between the simpleFoam and pimpleFoam solvers for the symmetric Y-Junction with a hypoplastic LPA, assuming steady flow. The length difference of a localised recirculation area at the entrance of the outer wall of the LPA was $10 \%$, while for the recirculation zone in the RPA, the difference was $2.7 \%$.

\section{Results}

The results of our study are presented in a series of figures that explore the effects of different geometric features on the heamodynamics of the pulmonary bifurcation (Fig. 3-6), and, the effect of boundary conditions on velocity and WSS in the symmetric Y-Junction, with and without a hypoplastic LPA (Fig. 7-9). The geometries reflect normal and abnormal cases of congenital heart patients, as described in Section 2.1 (Table 1).

\subsection{Effect of bifurcation geometry \\ 3.1.1. Contours of velocity magnitude}

Contours of non-dimensional velocity magnitude are presented in Fig. 3 for a segment of each of the nine bifurcation models described in Section 2.1 (Fig. 1). Due to the parabolic inlet profile, the highest values of velocity in the MPA were observed along the centreline and the lowest were found adjacent to the walls, for all models. For the geometries of Fig. 3 i-iv, 3 vi-viii, flow entering the bifurcation experienced an abrupt deceleration, with the maximum axial velocity value dropping rapidly, until becoming instantaneously zero when impinged on the wall (stagnation point, indicated with the letter S in Fig. 3). As a result, flow in the daughter branches was shifted towards the inner walls, with the highest values of velocity occurring adjacent to it, while extended low-velocity regions developed along the outer walls of the bifurcation.

The most substantial differences among the various models were observed for those with an LPA local stenosis (Fig. 3 vii-viii) or hypoplastic LPA (Fig. 3 v, 3 ix). For the models with local constriction (Fig. 3 vii-viii), the highest velocities were found inside the stenosis where the diameter of the artery was halved. Downstream of the stenosis, an extended region of low velocity values developed near the outer wall of the LPA, with an additional small-scale low-velocity region occurring at the inner wall of the model with symmetric narrowing (Fig. 3 viii). No substantial changes were noticed in the RPA velocity distribution of these models when compared with the symmetric non-stenotic model (Fig. 
3 vi). In contrast, for hypoplastic models (Fig. 3 v, 3 ix), where the LPA diameter was also halved, the highest velocities were observed in the RPA while very low velocities were exhibited in the hypoplastic LPA. The velocity distribution in these models deviated significantly from their respective non-stenotic symmetric and asymmetric models, and an altered flow split between the daughter branches was apparent.

The stagnation point, $\mathrm{S}$, was further identified for all models. It was located along the centreline of the MPA for the T-Junction (Fig. 3 i), the symmetric Y-Junction (Fig. 3 vi), and the asymmetric YJunction with non-common branch origins (Fig. 3 iv). For the remaining models, most stagnation points were found shifted away from the MPA centreline towards the RPA (denoted as $\mathrm{S}_{\mathrm{R}}$ in Fig. 3), by 0.77 $\mathrm{cm}(\sim 0.3 \mathrm{D})$ and $0.47 \mathrm{~cm}(\sim 0.18 \mathrm{D})$, respectively, for the asymmetric Y-Junctions of Fig. 3 ii-iii; 0.96 $\mathrm{cm}(\sim 0.37 \mathrm{D})$ for the asymmetric Y-Junction with hypoplastic LPA (Fig. $3 \mathrm{v}) ; 0.20 \mathrm{~cm}(\sim 0.077 \mathrm{D})$ for the symmetric Y-Junction with local symmetric stenosis (Fig. 3 viii); and $0.22 \mathrm{~cm}(\sim 0.085 \mathrm{D})$ for the hypoplastic LPA model (Fig. 3 ix). The only stagnation point found towards the LPA (denoted as $\mathrm{S}_{\mathrm{L}}$ ) was for the local asymmetric stenotic model (Fig. 3 vii), shifted by $0.075 \mathrm{~cm}(\sim 0.029 \mathrm{D})$ from the MPA centreline. The results indicate that the local geometric features of the pulmonary bifurcation can alter the stagnation point where fluid division takes place.

\subsubsection{Velocity profiles and flow splits}

In addition to the velocity contours, Fig. 4 presents non-dimensional velocity profiles at a distance of $3 \mathrm{~cm}(\sim 1.15 \mathrm{D})$ from the origin of the branches, for each of the models. As described, for most geometries (Fig. 4 i-iv, 4 vi-viii), flow velocities downstream of the bifurcation were skewed towards the inner walls of the daughter branches and away from the outer walls, where reverse (retrograde) flow was observed. However, the shape and extreme values of the velocity profiles exhibited differences in each of the LPA and RPA branches, as seen on the right side of Fig. 4. With the exception of the axisymmetric models (Fig. 4 i, 4 vi), peak velocities in the RPA were in general closer to the inner wall, compared to peak velocities in the LPA. The highest peak LPA velocity values were found for the symmetric models with local LPA stenosis (Fig. 4 vii-viii), which is consistent with the velocity increase due to the local decrease in the branch diameter. The highest peak RPA values were calculated for the models with hypoplastic LPA (Fig. 4 v, 4 ix).

In the asymmetric model of Fig. 4 ii, the velocity profile in the RPA exhibited the most extended reverse flow along the outer wall. A smaller extent in the reverse flow in the RPA was noticed for the models of Fig. 4 i, 4 iii-iv, 4 vi-viii, with the shortest of all for models of Fig. 4 v, 4 ix. The most extended reverse flow in the LPA branch was observed in the model with asymmetric local stenosis (Fig. 4 vii). Similar LPA flow reversal was seen in all other models, with the exception of the hypoplastic LPA geometries (Fig. 4 v-ix) where there was no reversal of flow in LPA. In the model with symmetric stenosis (Fig. 3 viii), flow was also reversed near the inner wall of the LPA.

As seen, the geometries with hypoplastic LPA (Fig. 4 v, 4 ix) had the biggest deviation in the velocity profiles in both daughter branches, as compared to all other models. There was a considerable increase in the RPA velocity in these cases, with a concomitant decrease in the LPA velocity. These profiles indicate that the flow was diverted primarily towards the RPA. To examine this further, the integrals of the velocity profiles were calculated to evaluate the flow rate in the MPA, RPA, and LPA. With the exception of the two models with hypoplastic LPA (Fig. 4 v, 4 ix), all other models (Fig. 4 iiv, 4 vi-viii) exhibited an equal flow rate in the right and left arterial branches (50:50\% for $\left.\mathrm{Q}_{\mathrm{LPA}}: \mathrm{Q}_{\mathrm{RPA}}\right)$, as measured at a distance of $3 \mathrm{~cm}(1.15 D)$ from the branch origin. However, a flow split ratio of approximately 12:88\% ( $\left.\mathrm{Q}_{\mathrm{LPA}}: \mathrm{Q}_{\mathrm{RPA}}\right)$ was estimated for both hypoplastic models (Fig. $4 \mathrm{v}, 4 \mathrm{ix}$ ), demonstrating that halving the diameter of one of the branches can significantly affect the distribution of flow in the pulmonary bifurcation. 


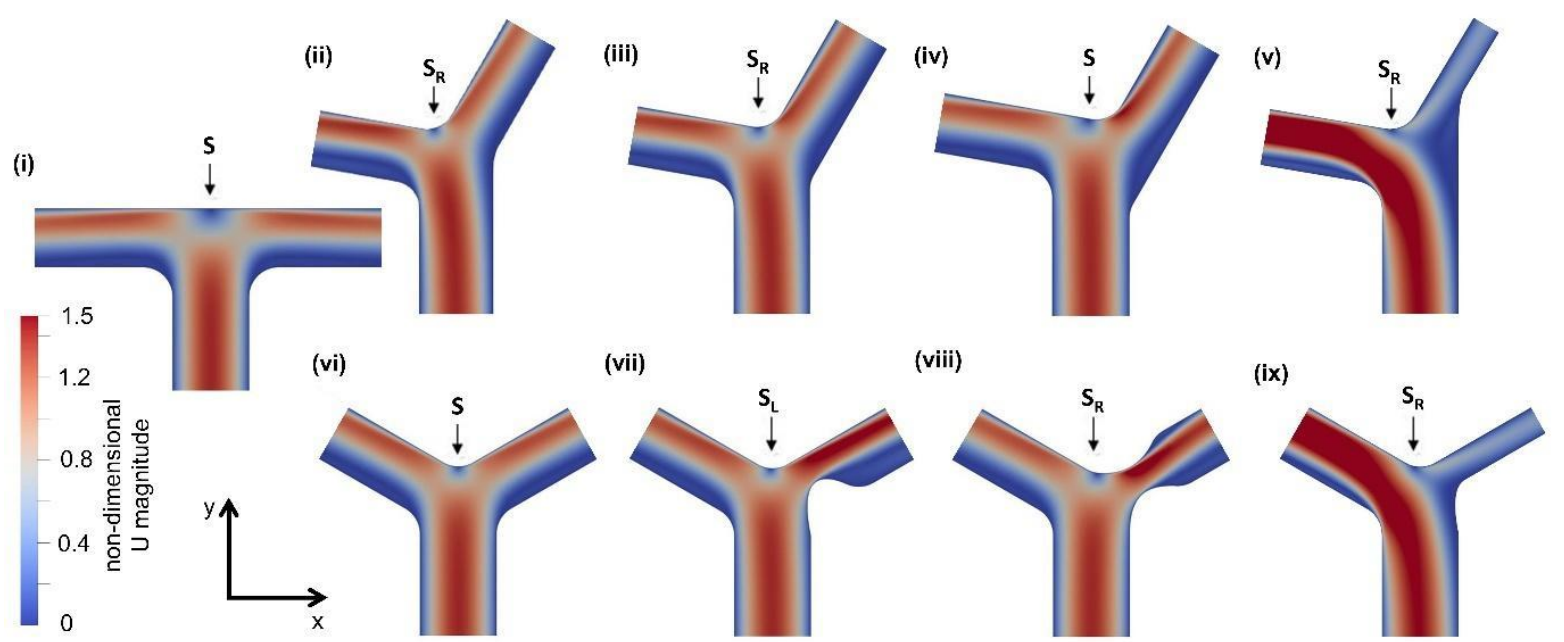

Fig. 3: Contours of non-dimensionalised velocity magnitude in models of the pulmonary bifurcation: (i) T-Junction; (ii)-(v) asymmetric Y-Junction with (iii)-(iv) different branch origins and (v) hypoplastic LPA; (vi-ix) symmetric Y-Junction (vi) without and (vii)-(viii) with local stenosis or (ix) hypoplastic LPA. Steady Newtonian flow, Re=650. The stagnation point on the MPA centreline is indicated with the letter $S$; or $S_{R}$ and $S_{L}$ if deviated towards the RPA and LPA, respectively.

(a)

(i)

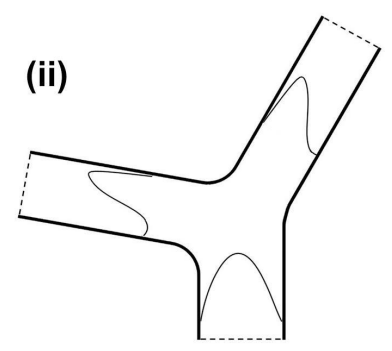

(iii)

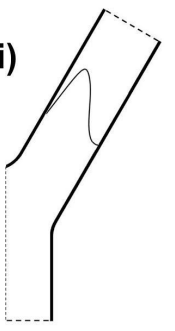

(iv)

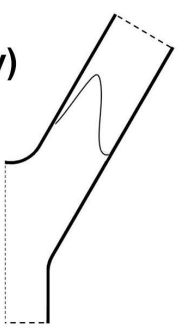

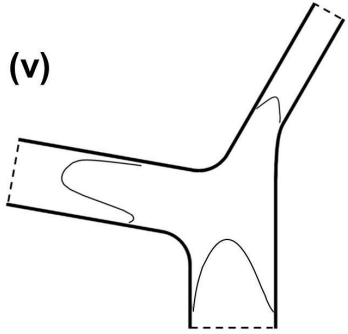

(vii) (viii) (ix)

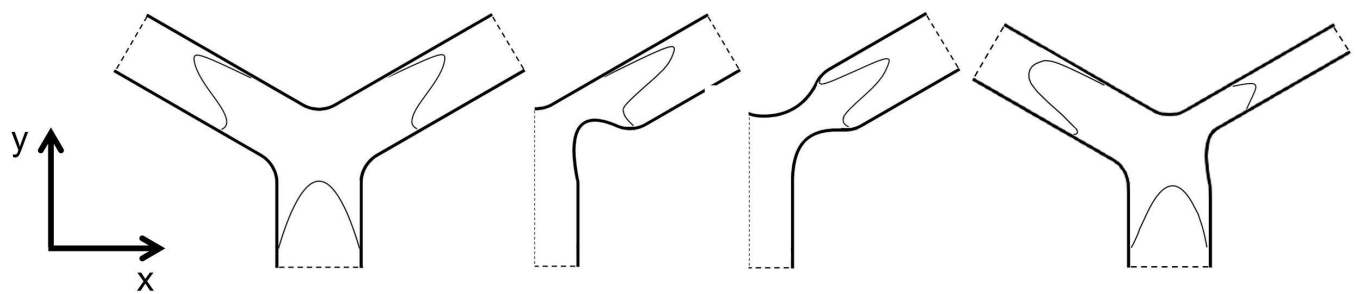

(b)
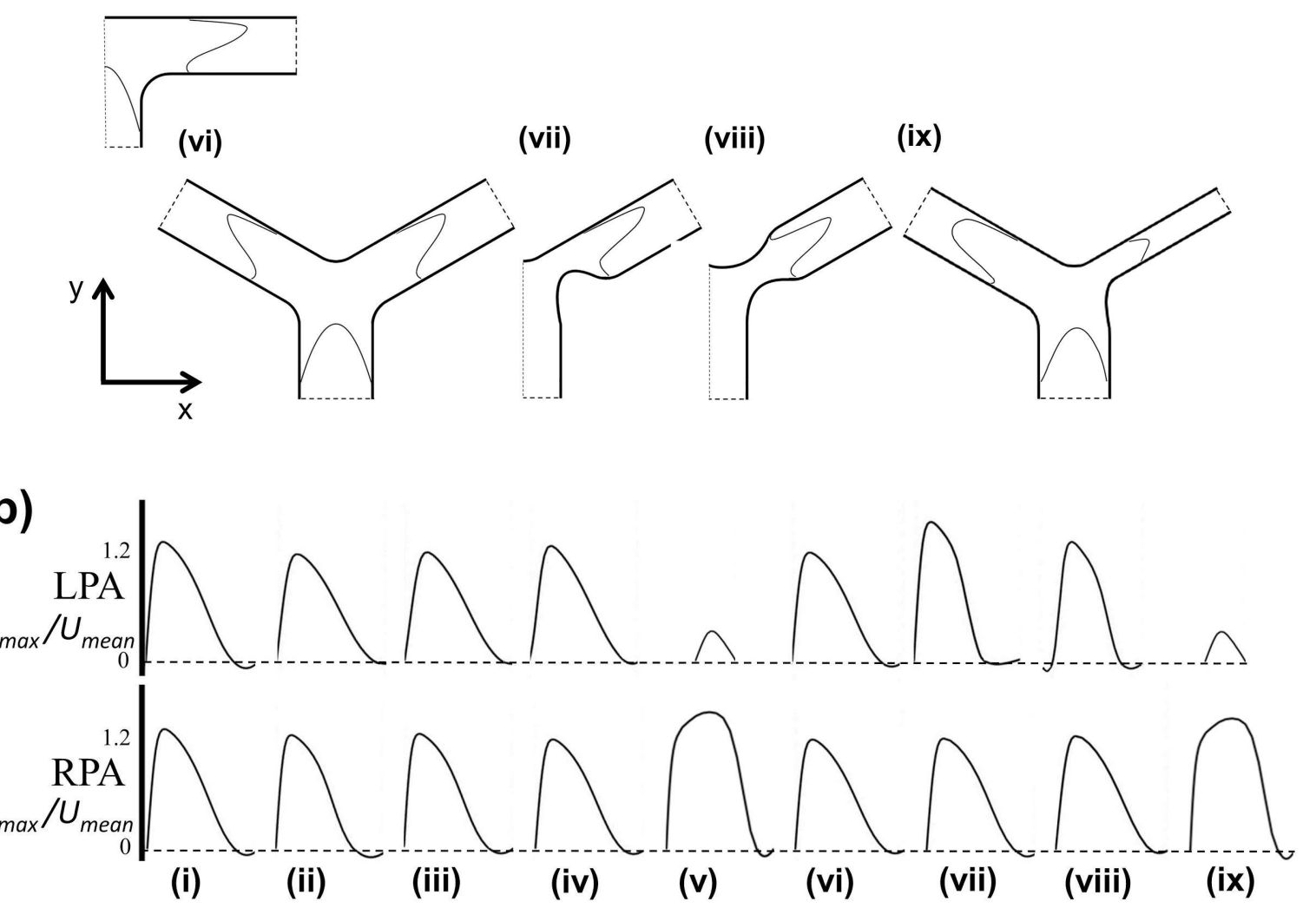

Fig. 4: Velocity profiles at a distance of $3 \mathrm{~cm}(\sim 1.15 D)$ from each branch origin presented (a) on top of each geometry and (b) placed comparatively next to each other. Dashed lines indicate cropped branch ends and symmetry planes (a) or the position of zero non-dimensionalised velocity (b). (i) T-Junction; (ii)-(v) asymmetric Y-Junction, with the different branch 
origins or hypoplastic LPA; (vi)-(xi) symmetric Y-Junction, without or with local stenosis or hypoplastic LPA. Steady Newtonian flow, $R e=650$.

\subsubsection{Velocity streamlines}

Fig. 5 presents velocity streamlines in the nine geometries of Fig. 1. Arrows along the streamlines indicate the direction of fluid flow from the main pulmonary artery (MPA) to the daughter branches. It is evident that recirculation zones develop in all models, independent of the branching angle for RPA or LPA, that is, at $90^{\circ}$ (Fig. 5 i), $100^{\circ}$ (Fig. 5 ii-iv), $120^{\circ}$ (Fig. 5 vi-ix), and $150^{\circ}$ (Fig. 5 ii-iv). In the asymmetric Y-Junction of Fig. 5 ii, the $100^{\circ}$ angle between the MPA and RPA $\left(\theta_{l}\right)$ had an extended recirculation area, while only a small region of recirculating fluid was observed for the $150^{\circ}$ MPA-LPA angle $\left(\theta_{2}\right)$. For the same branching angles but with different origins (Fig. 5 iii-iv), the recirculation zones at the RPA $\left(\theta_{1}=100^{\circ}\right)$ were reduced, while at the entrance of the LPA $\left(\theta_{2}=150^{\circ}\right)$ they were extended considerably.

Elongated recirculation regions were also observed in the symmetric models with local stenosis (Fig. 5 vii, 5 viii). In Fig. 5 vii, recirculation of flow occurred downstream of the asymmetric stenosis, along the outer wall of the bifurcation, while similar zones downstream of the symmetric stenosis in Fig. 5 viii were seen adjacent to both the outer and inner walls of the arterial branch. For the two hypoplastic models (Fig. 5 v, 5 ix), flow recirculation occurred at the entrance to the RPA and immediately before entering the left pulmonary artery. This zone was more extended in the asymmetric model (Fig. $5 \mathrm{v}$ ). For all models, there is a region of accelerating flow downstream of the stagnation point (S, Fig. 3), which becomes more pronounced within the stenosis for the models of Fig. 5 vii, viii.

Flow separation is a common phenomenon in bifurcations and occurs when the fluid velocity cannot expand with the geometry or when an adverse pressure gradient is present causing convection on the fluid laminae. Fluid particles in these cases are captured within the recirculation area and cannot re-enter the bulk flow due to their incapacity to overcome the flowing fluid pressure [12]. The results of Fig. 5 indicate that the upstream separation point, where recirculation zones start to develop, was at the same position for all non-stenotic models, that is, at the entrance of the bifurcation. However, the extent of these zones appears to be affected by the branch origin, resulting in different locations of the downstream flow reattachment points. Increased regions of recirculation cannot be correlated with an increase in the branching angle, as previous studies have indicated [3, 20]. The models with local stenosis (Fig. 5vii-5viii) and hypoplastic LPA (Fig. 5 v, 5 ix) have zones developed downstream of the stenosis or upstream of the entrance to the left pulmonary branch, respectively. Local stenosis appeared to cause extension of recirculation zones downstream of the stenosis (Fig. 5 vii, 5 viii), while the hypoplastic models cannot be correlated with increased regions of recirculation further downstream within the LPA (Fig. 5 v, 5 ix).

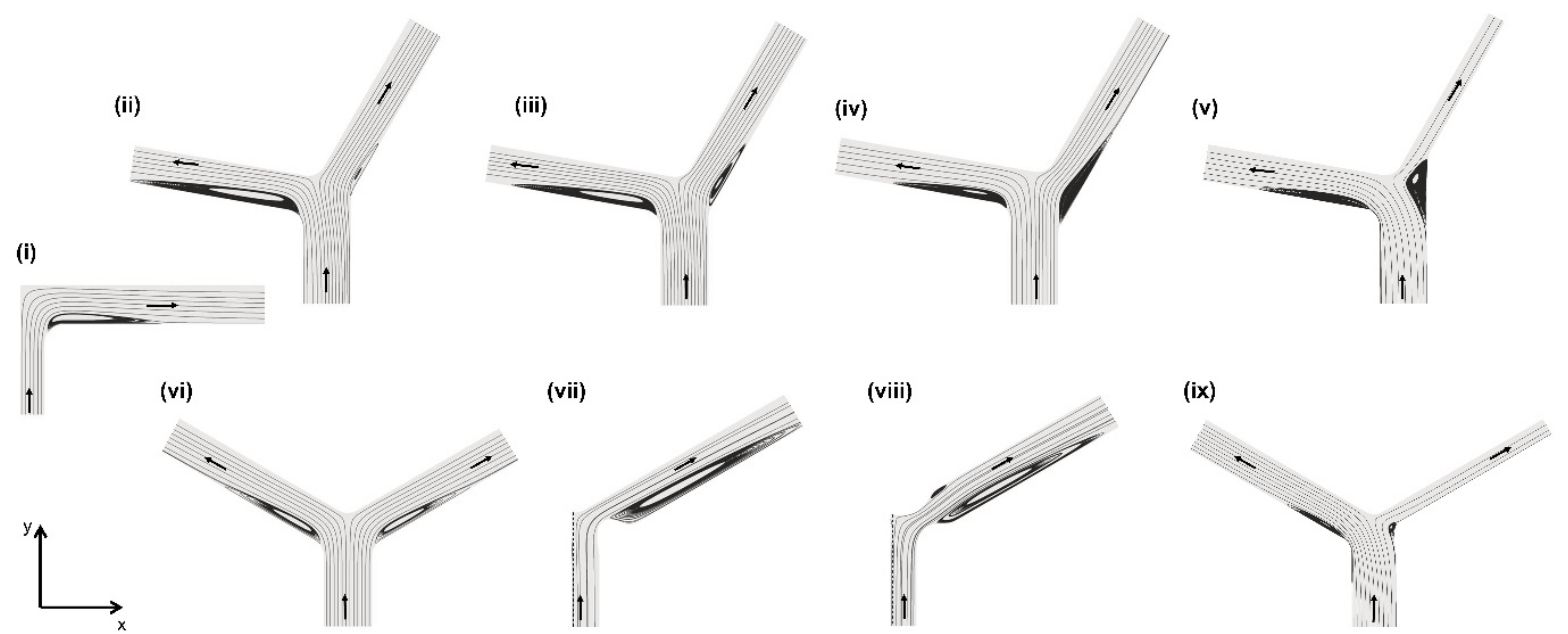

Fig. 5: Velocity streamlines in the models of Fig. 1: (i) T-Junction; (ii)-(iv) asymmetric Y-Junction models with different branch origins and (v) with hypoplastic LPA; (vi) symmetric Y-Junction; (vii)-(viii) models with local stenosis; (ix) model with hypoplastic LPA. Steady Newtonian flow, Re=650. 


\subsubsection{Wall shear stress profiles}

Wall shear stress distribution along the inner wall of the bifurcation is presented in Fig. 6, nondimensionalised according to the WSS value at the MPA inlet, as described in Section 2.4. The zero position in the abscissae of these plots signifies the stagnation point $\left(S, S_{R}\right.$, or $S_{L}$, see Fig. 2), while normalisation of the distance $(l)$ from point $S$ along the inner wall is based on the MPA diameter, D.

Examining the effect of branching angle (Fig. 6a), it appears that while the symmetric YJunction (dotted line in Fig. 6a) and the T-Junction model (dash line in Fig. 6a) presented symmetric WSS profiles on both branches, the asymmetric Y-Junction (solid line in Fig. 6a) obtained significantly increased WSS values towards the LPA $\left(\theta_{2}=150^{\circ}\right)$. The location of the maximum WSS values for each model was also different, with that for the T-Junction being the furthest away from the stagnation point.

Displacing the origins of one or both branches (of the same angle) caused a further increase of non-dimensionalised WSS values in the LPA of the asymmetric Y-Junctions and a simultaneous WSS reduction in the RPA (Fig. 6b). In addition, the location of the highest WSS values for each model moved further away from the stagnation point in both branch directions. The geometry of Fig. 3 iv resulted in a well-defined high WSS region at the short distance from the stagnation point towards the LPA, taking on very low values further downstream.

For the models with local stenosis (Fig. 6c), there was a clear increase at the WSS values along the inner wall of the LPA, without a strong influence to the WSS in the RPA. This was particularly evident for the symmetric stenotic model which exhibited a very different WSS distribution in the LPA.

Interestingly, for the geometries with hypoplastic LPA (Fig. 6d), the WSS in the LPA dropped to approximately the same values irrespective of the branching angle of the LPA bifurcation $\left(120^{\circ}\right.$ or $150^{\circ}$ ). WSS in the RPA also decreased but obtained different values for the asymmetric and symmetric models, and the peak WSS was shifted away from the stagnation point; these profiles resembled somewhat the WSS distribution at the RPA of the T-Junction (dash line in Fig. 6a).
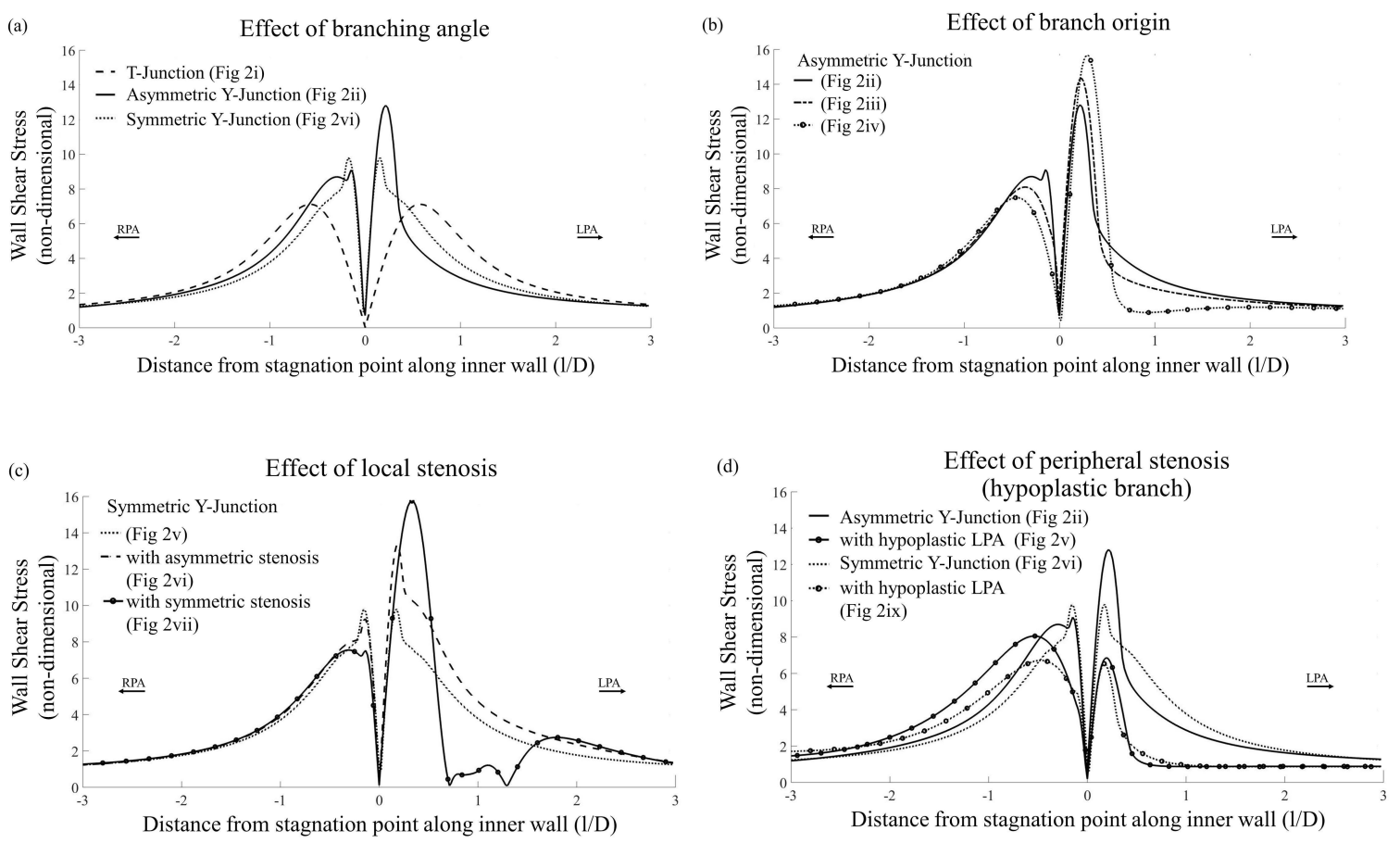

Fig. 6: Non-dimensionalised wall shear stress (WSS) distribution along the inner wall of the models of Fig. 2, for steady Newtonian flow, $R e=650$ : (a) models with different branching angles; (b) asymmetric models with different branch origins; (c) symmetric Y-Junction without and with local stenosis; (d) asymmetric and symmetric Y-Junctions without and with hypoplastic LPA. The zero position in the abscissae signifies the stagnation point $\left(S, S_{R}\right.$, or $S_{L}$, see Fig. 2).

\subsection{Effect of outlet boundary conditions}




\section{Prescribed pressure outlet conditions}

The effect of the pressure boundary condition at the branch outlets was tested in two ways (Fig. 7). Flow was diverted towards the LPA branch where higher velocities were observed for the extended RPA model (Fig. 7a-i); in this model, lower velocities were noticed in the RPA with strong recirculation zones. The opposite was observed in the models with a normalised branch pressure difference $\left(\frac{|\Delta P|}{0.5 \rho U^{2}}\right)$ of 0.026 (for $\mathrm{P}_{\mathrm{LPA}}>\mathrm{P}_{\mathrm{RPA}}$, Fig. 7a-ii) and 0.015 (also $\mathrm{P}_{\mathrm{LPA}}>\mathrm{P}_{\mathrm{RPA}}$, Fig. 7a-iii), where flow diverted towards the right branch. The streamlines of the model in Fig. 7a-iii present decreased recirculation zones when compared to Fig. 7a-ii both at RPA and LPA.

Velocity profiles at a distance of $3 \mathrm{~cm}(1.15 D)$ from the branch origin were also plotted for the RPA and LPA branches (Fig. 7b), confirming the above observations. A slight increase in the forward velocity in the LPA was seen in the case of Fig. 7a-iii, as compared to Fig. 7a-ii. The velocity profiles for these models indicate variations in the branch flow splits, with the flow diverted towards the LPA (Fig. 7a-i) and towards the RPA (Fig. 7a-ii, iii). The flow rates in the LPA and RPA were calculated for all models and flow split ratios were found approximately 67:33\% for Fig. 7a-i, 33:67\% for Fig. 7a-ii and $40: 60 \%$ for Fig. 7a-iii $\left(\mathrm{Q}_{\mathrm{LPA}}: \mathrm{Q}_{\mathrm{RPA}}\right)$. As expected, the results indicate that the pressure boundary condition can alter the flow split in the pulmonary bifurcation from the 50:50\% ratio in the daughter branches.

The stagnation point was further identified for these three models. For the geometries of Fig. $7 \mathrm{a}-\mathrm{i}$ and $7 \mathrm{a}-\mathrm{ii}$, the stagnation point was found $0.12 \mathrm{~cm}(0.046 \mathrm{D})$ towards the LPA and the RPA, respectively, while for the model of Fig. 7a-iii, the stagnation point was located $0.0075 \mathrm{~cm}(0.0029 D)$ towards the RPA.

Non-dimensionalised wall shear stress profiles are presented in Fig. $7 \mathrm{c}$ and are compared with the WSS obtained for the symmetric geometry of Fig. 2 vi with zero pressure at both branch outlets (dash line in Fig. 6a, repeated as solid line in Fig. 7c). A slight increase is observed in the WSS developed at the inner wall of the RPA, with a simultaneous WSS reduction at the LPA wall for the model of Fig. 7a-i. Accordingly, a decrease was observed in the WSS along the inner wall of the RPA of the models with the pressure difference at the branch outlets (Fig. 7a-ii, iii). 
(a)

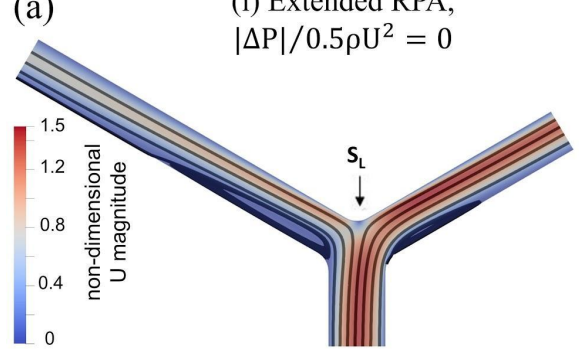

$U_{\text {max }} / U_{\text {mean }}^{1.2}$ (i) Extended RPA,

$|\Delta \mathrm{P}| / 0.5 \rho \mathrm{U}^{2}=0$ (ii) $\mathrm{P}_{\mathrm{LPA}}>\mathrm{P}_{\mathrm{RPA}}$,

$|\Delta \mathrm{P}| / 0.5 \rho \mathrm{U}^{2}=0.026$ (iii) $\mathrm{P}_{\mathrm{LPA}}>\mathrm{P}_{\mathrm{RPA}}$,

$|\Delta \mathrm{P}| / 0.5 \rho \mathrm{U}^{2}=0.015$

(c)
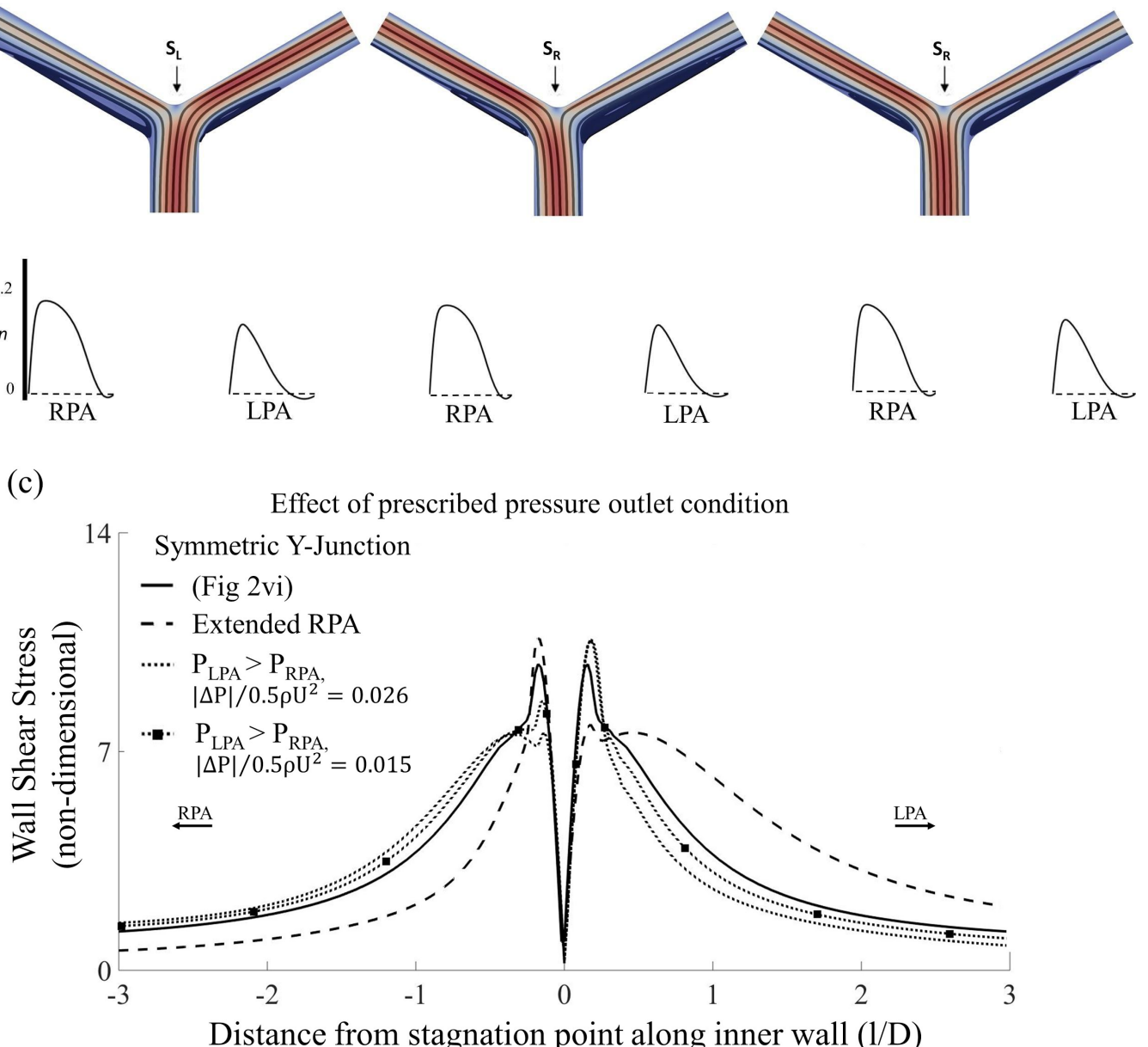

Fig. 7: Effect of pressure boundary condition at branch outlet. (a) Non-dimensionalised velocity contours overlaid by streamlines. (b) velocity profiles along the RPA and LPA. (c) Non-dimensionalised WSS distribution along the inner wall for the geometry of Fig. $2 f$ (solid line) and model with (i) an extended right pulmonary branch with $\frac{|\Delta P|}{0.5 \rho U^{2}}=0$, (ii) $\frac{|\Delta P|}{0.5 \rho U^{2}}=0.026$ with $P_{L P A}>P_{R P A}$ and (iii) $\frac{|\triangle P|}{0.5 \rho U^{2}}=0.015$ with $P_{L P A}<P_{R P A}$. Steady Newtonian flow, $R e=650$.

\section{Prescribed flow splits}

Contours of non-dimensional velocity magnitude are presented in Fig. 8a-i $\left(\mathrm{Q}_{\mathrm{LPA}}: \mathrm{Q}_{\mathrm{RPA}}=\right.$ 11:89\%) and Fig. 8a-ii (Q $\left.\mathrm{LPA}_{\mathrm{LP}} \mathrm{Q}_{\mathrm{RPA}}=20: 80 \%\right)$ for Murray's Law and the "outflow splitting" method (Eqs. 5 and 6, Section 2.3), respectively. As expected, the results are comparable with those of Fig. 3 ix, with the majority of the flow diverted to the RPA. The stagnation point, $\mathrm{S}$, was found $0.22 \mathrm{~cm}(\sim 0.085 D)$ and $0.17 \mathrm{~cm}(\sim 0.065 D)$ towards the RPA, for the models of Fig. 8a-i and 8a-ii, respectively. The nondimensionalised WSS distributions along the inner wall of the bifurcation are shown in Fig. 8b. With Murray's Law (Fig. 8a-i), the WSS is very similar to that with zero-pressure boundary condition (Fig 
6d), with slightly lower peak WSS value in the LPA. WSS is increased in both branches for the model with the "outflow splitting" method (Fig. 8a-ii), which is more prominent in the LPA (Fig. 8b).
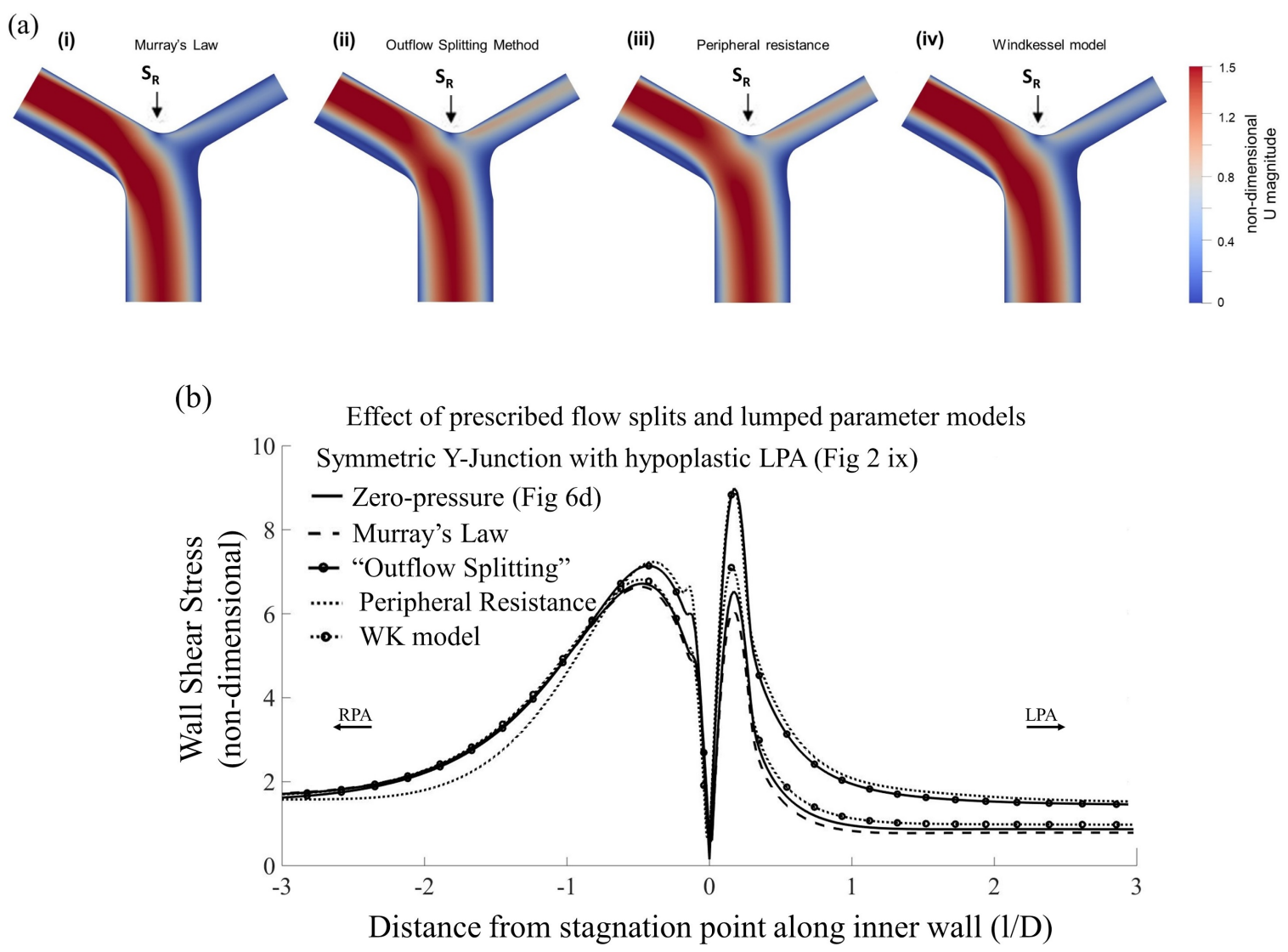

Fig. 8: Effect of outlet boundary conditions. (a) Non-dimensionalised velocity contours. (b) Non-dimensionalised WSS distribution along the inner wall for the geometry of Fig. 2ix (solid line) with (i)-(ii) flow split dictated by (i) Murray's Law $\left(Q_{L P A}: Q_{R P A}\right.$ is 11:89\%) and (ii) "outflow splitting” method ( $Q_{L P A}: Q_{R P A}$ is 20:80\%), and (iii)-(iv) coupled lumped parameter models with, (iii) a peripheral resistance ( $Q_{L P A}: Q_{R P A}$ is $\left.22: 78 \%\right)$ and (iv) a three-element Windkessel (WK) model $\left(Q_{L P A}: Q_{R P A}\right.$ is 14:86\%). Steady Newtonian flow, $R e=650$.

\section{Coupled Lumped Parameter Models}

Fig. 8a also presents non-dimensionalised velocity contours for a peripheral resistance boundary condition (Fig. 8a-iii, $\mathrm{Q}_{\mathrm{LPA}}: \mathrm{Q}_{\mathrm{RPA}}=22: 78 \%$ ), and a three-element Windkessel model (Fig. 8aiv, $Q_{L P A}: Q_{R P A}=14: 86 \%$ ), for steady-state inlet flow. Similar to cases Fig. 8a-i, a-ii and Fig. 3ix, most of the flow is directed towards the RPA. For both cases, the stagnation point is shifted towards the RPA by $0.12 \mathrm{~cm}(\sim 0.046 D)$ (Fig. 8a-iii) and $0.17 \mathrm{~cm}(\sim 0.065 D)$ (Fig. 8a-iv). The WSS for the simulation with the peripheral resistance (Fig. 8a-iii) exhibits an increase in both daughter branches, similar to Fig. 8a-ii. With the Windkessel model assumption (Fig. 8a-iv), the WSS resembles that of Fig. 6d, but with a small increase in the WSS in the LPA (Fig. 8b).

Instantaneous non-dimensionalised velocity contours and wall shear stress distribution are presented in Fig 9a for the model of Fig. 3 ix with time-dependent sinusoidal inlet profile. At maximum acceleration, the WSS and velocity magnitude take the highest values during the cycle (Fig. 9a-i). During deceleration, both the velocity and WSS decrease until they reach the lowest values, at maximum deceleration (Fig. 9a-ii, a-iii). The peak WSS is higher along the inner wall of the LPA, except at the middle of acceleration (Fig. 9a-iv). The flow splits for each of the four time points were $\mathrm{Q}_{\mathrm{LPA}}: \mathrm{Q}_{\mathrm{RPA}}=28: 72 \%$ (Fig. 9a-i), 22:78\% (Fig. 9a-ii), 51:49\% (Fig. 9a-iii), and 22:78\% (Fig. 9a-iv). An average flow split of $\mathrm{Q}_{\mathrm{LPA}}: \mathrm{Q}_{\mathrm{RPA}}=22: 78 \%$ was calculated over one cycle. The stagnation point was also identified, and was found shifted towards the RPA by $0.075 \mathrm{~cm}(\sim 0.028 D), 0.22 \mathrm{~cm}(\sim 0.085 D), 0.54$ $\mathrm{cm}(\sim 0.21 D)$, and $0.12 \mathrm{~cm}(\sim 0.046 D)$ for the four time-points (Fig. 9ai-iv), respectively. The time- 
averaged WSS for the unsteady flow is compared with the steady flow in Fig. 9b. A similar pattern in the wall shear stress distribution is observed, but with slightly higher values for the TAWSS.

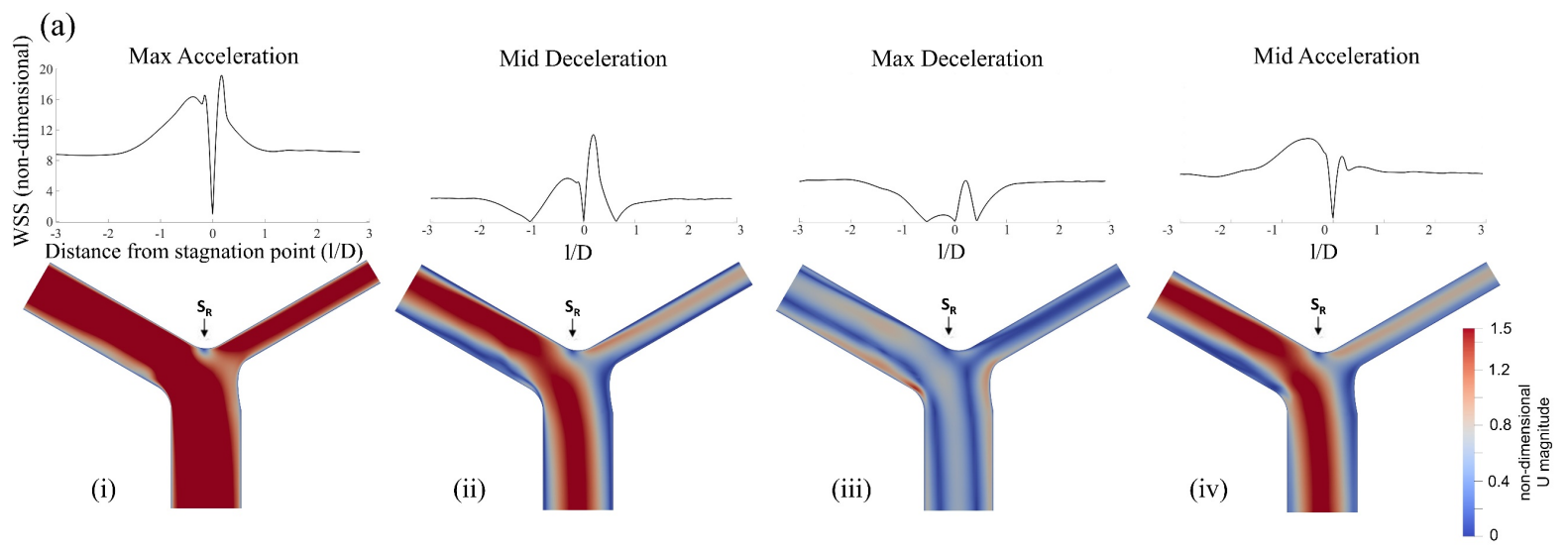

(b)

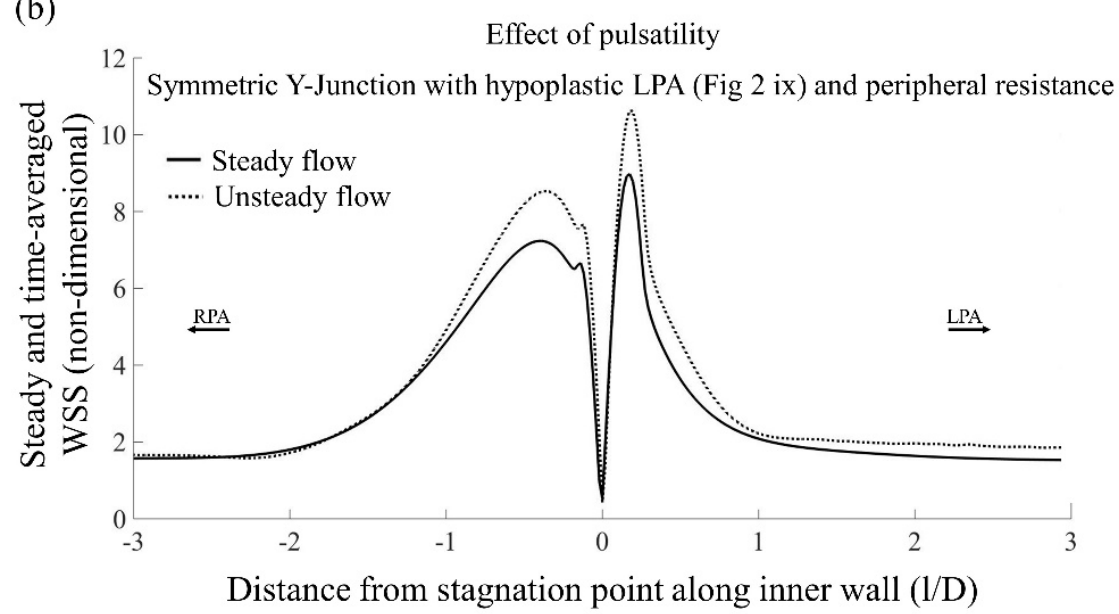

Fig. 9: Effect of pulsatility in the symmetric Y-Junction with hypoplastic LPA and the peripheral resistance boundary condition. (a) Instantaneous non-dimensionalised velocity contours and WSS distribution along the inner wall, at maximum acceleration, middle of deceleration, maximum deceleration and middle of acceleration of a sinusoidal waveform (Eq. 4). (b) Steady and time-averaged non-dimensional wall shear stress distribution for steady and unsteady flow. Newtonian flow, mean $R e=650$.

\subsection{Sensitivity analysis}

Further results from a sensitivity analysis of the effects of various different boundary conditions (Section 2.5) are presented in the Supplementary Material. The findings highlight some additional computational aspects that may influence the flow and WSS distribution in the pulmonary bifurcation, providing, more importantly, a comparison between a two-dimensional and a three-dimensional bifurcation, for both steady and unsteady cases. Moreover, this analysis takes into account the rheological properties of blood, through the use of different non-Newtonian models, and their role on fluid flow and WSS in the pulmonary arteries.

\section{Discussion}

Adult patients with repaired tetralogy of Fallot constitute an increasing population that require regular monitoring and specialised care due to unpredictable and long-term complications. Pulmonary regurgitation and left pulmonary kinking are the most frequent causes for re-intervention, commonly treated through pulmonary valve replacement [6]. Analysis of the blood flow development in the pulmonary arteries of these patients is important and may improve clinical evaluation and decision- 
making for PVR [18-19, 22]. To that end, the focus of the present study was to elucidate computationally the effects of different geometric parameters and outlet boundary conditions on the haemodynamics in simplified models of the pulmonary bifurcation.

\subsection{Simplifying assumptions}

Arguably, the most important simplification of this study was the assumption of 2D geometries for the pulmonary bifurcation. This choice was made to facilitate an extensive investigation of different geometric parameters and boundary conditions, and was supported by a comparison with a threedimensional geometry demonstrating qualitative similarities in the shape of the WSS distribution along the inner wall of the bifurcation, for both 2D and 3D models, despite the quantitative differences (see Supplementary Material). We, therefore, explain the use of the simplified models, since the aim of this work was to capture the main characteristics of the flow and WSS distribution in the pulmonary bifurcations under a range of different conditions. This would be difficult to perform with threedimensional patient-specific geometries since the parametric variation would be more complex to define.

Another important simplification was the assumption of steady flow. Some aspects of unsteadiness can be considered negligible for large vessels [39, 40], particularly since physiological fluctuations in mean velocity during the cardiac cycle are adequately slow to assume quasi-steady flow. This was confirmed here by the similarity in WSS values between steady and unsteady sinusoidal flows, for both 2D and 3D geometries (Fig. 9b and Supplementary Material). Finally, the assumption of rigid walls is another assumption that may influence the computational results and will be considered in future work. Nevertheless, wall compliance has been found to have a minimal effect on the overall flow [16]. Indeed, the radial aortic wall deformation is also relatively small during the cardiac cycle compared with the axial unsteady flow into the pulmonary branches [41], and could be neglected. It is, therefore, reasonable to expect that the work presented here provides a good indication of the effects of the same or similar conditions in the respective $3 \mathrm{D}$ models and captures qualitatively the characteristics of 3D unsteady flow.

\subsection{Comparison with other works}

Previous studies have investigated the effect of angle in the pulmonary bifurcation and found that recirculation zones develop for branch angles close to or equal to $100^{\circ}$ [3], but not for an angle of $150^{\circ}$ [19]. This is in agreement with our results for branch angles $90^{\circ}, 100^{\circ}$ and $150^{\circ}$ (Fig. 5 i, v, ii, respectively); however, Fig. 5 iii, iv demonstrate that new recirculation zones develop simply by the displacement of the branch origins, for the same branch angle of $150^{\circ}$. Our results, therefore, clarify that increased recirculation cannot be correlated only with increased branching angle, and that further to the branch angle, the origin of the pulmonary branches is also important in analysing the local flow development. For the local stenotic models (Fig. 5 vii-viii), the flow separation, and the WSS profiles (Fig. 6c) obtained is in comparison to the study of Kanokjaruvijit et al. (2017) [42], where different types of stenotic bifurcations were investigated, and with Katritsis et al. (2010) [43]. Major flow separation was found to occur when the diameter inside the stenosis decreased to $50 \%$, and wall shear stress was increased inside the stenotic branches, followed by a rapid decrease downstream [42]. The contours of the non-dimensionalised velocity magnitude, as presented in Fig. 3, are also in agreement with those reported in similar studies [43]. In addition, our work is comparable to the work of Szymanski et al. (2008) [44], who observed a similar WSS pattern on the inner wall of a T-Junction, and analysed the importance of the stagnation point. Immediately downstream of the stagnation point, where the velocity is zero and the wall shear stress is very low, a region of high WSS was present, followed by low WSS values [44], similar to the results presented here. Our study, further, demonstrates that the location of flow division in bifurcating flows clearly influences the WSS distribution on the inner wall, as shown in Fig. 6, 7c, 8b, however no previous studies were found investigating this effect.

\subsection{Physiological, pathological, and clinical relevance}

It has been proposed that in the presence of pulmonary valve regurgitation, branch stenosis limits the flow to the corresponding lung and possibly aggravates the severity of PR [45]. In addition, differential reverse flow between the pulmonary branches (branch regurgitation) may be positively 
correlated with pulmonary vascular resistance [5]. Patients without pulmonary branch stenosis appear to have increased LPA regurgitation, while patients with stenosis or a hypoplastic branch are associated with increased retrograde flow in the larger artery and consequently increased pulmonary vascular resistance. Therefore, patients with stenosis or a hypoplastic branch and significantly differential branch regurgitation, are expected to have elevated pulmonary vascular resistance in the enlarged non-stenotic branch [5]. The percentage of diameter reduction also dictates the flow split between the two branch arteries. With the majority of flow diverted to the enlarged RPA, the differential backflow is expected to increase accordingly, along with the pulmonary vascular resistance and pressure in the non-stenotic branch, increasing the risk of pulmonary vascular diseases and hypertension during childhood. In general, reduced flow in the LPA is a clinical observation in many TOF patients, due to anatomical variations, which complicates flow in their pulmonary arteries [3]. Flow imbalance between the LPA and the RPA may also have several clinical consequences, leading to ventilation or perfusion mismatch, or adversely influence pulmonary microvascular growth if preserved for extended periods during childhood. We reflect on these views, by extending, in the following paragraphs, an analysis on the pressure ratios and flow splits in the models presented in this study.

\subsubsection{Pressure ratios}

The importance of appropriate pressure conditions in arterial models has been extensively discussed in the literature [46, 47]. In this study, we investigated the effect of different conditions at the branch outlets (Section 3.2.2), including the use of more realistic pressure values (Fig. 7, 8). These values fall within the range of pressures computationally calculated in the study of Arbia et al. (2014) [48], expressed relative to a reference pressure at the outlets of the models.

A more clinically-relevant way of understanding the pressure changes along the pulmonary bifurcation is through comparison of the pressure ratios between the two branches $\left(\mathrm{P}_{\mathrm{LPA}}: \mathrm{P}_{\mathrm{RPA}}\right)$ and between the daughter and parent vessels $\left(\mathrm{P}_{\mathrm{LPA}}: \mathrm{P}_{\mathrm{MPA}}\right.$ and $\left.\mathrm{P}_{\mathrm{RPA}}: \mathrm{P}_{\mathrm{MPA}}\right)$. For that, the static pressure within each vessel was measured at four locations along the centreline, at distances 4 to $7 \mathrm{~cm}(\sim 1.5 D$ to $\sim 2.7 D)$ from the branch origin, and at additional symmetrical locations on either side of the centreline, at 0.65 $\mathrm{cm}(0.25 D)$ from the walls of the MPA and $0.5 \mathrm{~cm}(\sim 0.2 D)$ from the walls of both the RPA and LPA (total of 12 points, as shown in Fig. 10a, overlaying pressure contours in the symmetric Y-Junction, non-dimensionalised by $0.5 \rho$ ). The mean arterial pressure ratios, along with the standard deviation, were then calculated based on the 12 measurements for each vessel. Table 2 provides the measured pressure ratios and standard deviation, while Fig. $10 \mathrm{~b}$ presents the $\mathrm{P}_{\mathrm{LPA}}: \mathrm{P}_{\mathrm{RPA}}$ and $\mathrm{P}_{\mathrm{LPA}}: \mathrm{P}_{\mathrm{MPA}}$ ratios, where 1 indicates equal pressure values.

For the symmetric T- and Y-Junctions, the left and right pulmonary branches have the same mean pressure, which is slightly higher than that in the MPA (cases (i) and (vi) in Table 2 and Fig. 10b). Introducing asymmetry in the bifurcation (asymmetric Y-Junction models (ii)-(v) in Table 2 and Fig. $10 \mathrm{~b}$ ), results in an increase in the mean pressure developed in the LPA with a concomitant decrease in the RPA, as compared to the pressure in the MPA; an exception to this is for the asymmetric model with both branch origins displaced (Table 2 (iv)) which exhibits almost the same mean pressure in both RPA and MPA. The asymmetric Y-Junction with hypoplastic LPA (Table 2 (v), Fig. 10b) has the highest mean pressure difference between the left and right pulmonary branches among all models, with the pressure in the LPA being clearly higher than that in the RPA. The symmetric models with local stenosis (Table 2 (vii)-(viii), Fig. 10b) exhibit a distinctive decrease in the mean LPA pressure, while the mean RPA pressure is very similar to that of the MPA. For the symmetric model with hypoplastic LPA (Table 2 (ix), Fig. 10b), the mean LPA pressure is elevated, like in the asymmetric model (Table 2 (v)), and is higher than the RPA pressure, which in turn is lower than that in the MPA. The opposite is found for the model with an extended RPA branch (Table 2 (x), Fig. 10b), where the mean LPA pressure is lower than the mean RPA and MPA pressures. For the models with the pressure difference at the outlets (Table 2 (xi)-(xii), Fig. 10b), the mean LPA pressures are higher than those in the RPA. Finally, for the four different outflow conditions tested in the symmetric Y-Junction with the hypoplastic LPA (Table 2 (xiii-xvi), Fig. 10b), the mean LPA pressure is elevated while the mean pressure in the RPA is reduced, similar to case (ix), Table 2. All pressure ratios for the model with the flow splits as predicted by the Murray's Law (Table 2 (xiii)) are almost identical to those of case (ix) and slightly different to case (xvi) with the coupled Windkessel model. Among the last four cases (xiii- 
$\mathrm{xvi}$ ), the model with the peripheral resistance at the branch outlets (Table 2 (xv)) has the largest deviation in the $P_{L P A}: P_{R P A}$ ratio from case (ix) in Table 2.

(a)
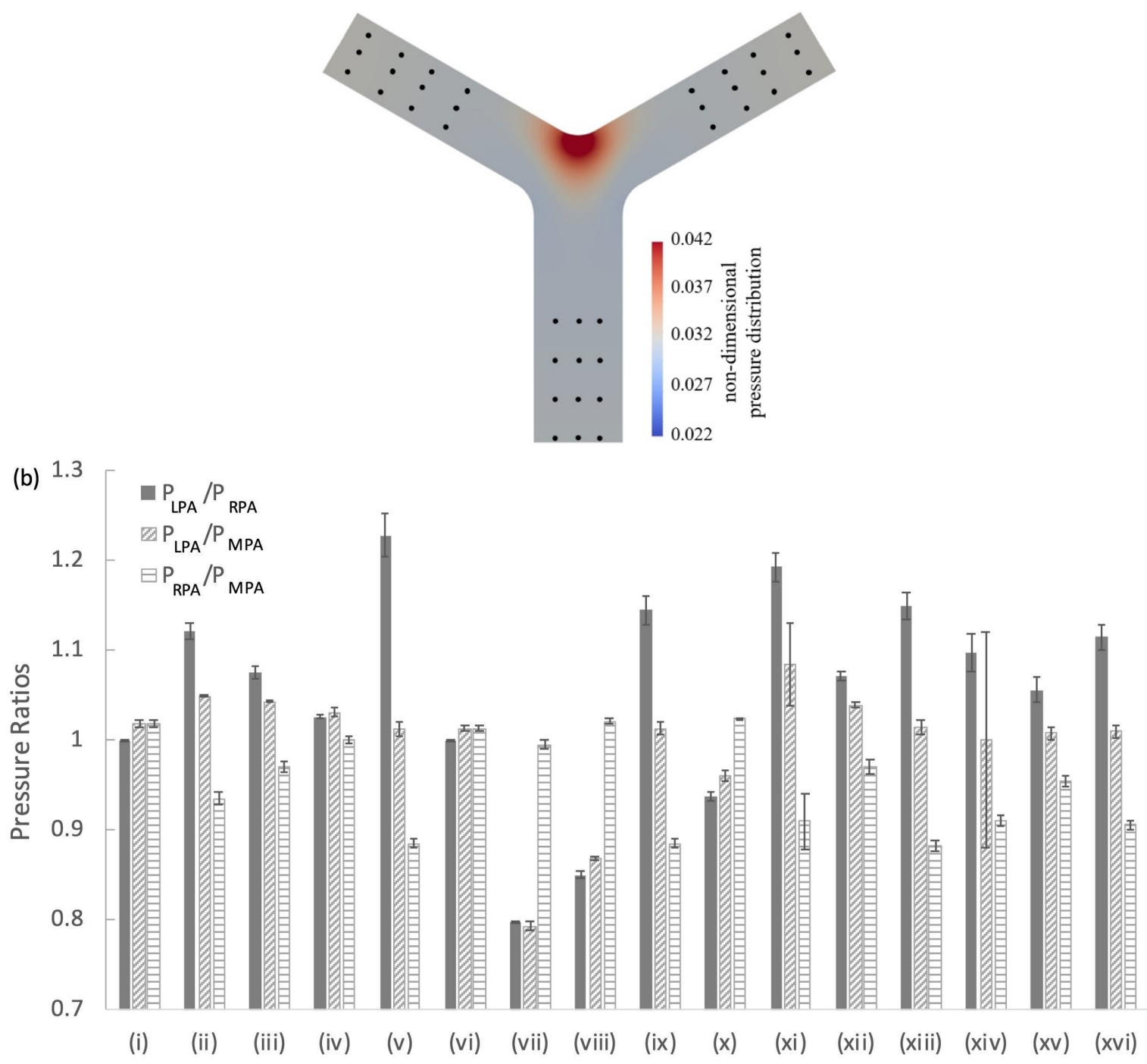

Figure 10: (a) Points where the pressure values were measured, overlaying contours of pressure in the symmetric Y-Junction. Pressure is depicted relative to the reference pressure at the outlets, and normalised by $0.5 \rho U^{2}$. (b) Pressure ratios for the models examined: (i) T-Junction (Fig. 2i); (ii)-(v) asymmetric Y-Junction models, with (ii) common origin (Fig. 2ii), (iii) one (Fig. 2iii) or (iv) both branch origins displaced (Fig. 2iv), or (v)hypoplastic LPA (Fig. 2v); (vi) symmetric Y-Junction (Fig. 2vi), with (vii) local asymmetric stenosis (Fig. 2vii), (viii) local symmetric stenosis (Fig. 2viii), or (ix) hypoplastic LPA (Fig. 2ix); (x) symmetric Y model with extended RPA (Fig. 7a-i); (xi)-(xii) symmetric Y model with $P_{L P A}<P_{R P A}$ and with $\frac{|\Delta P|}{0.5 \rho U^{2}}=$ 0.026 (Fig. 7a-ii), and with $\frac{|\Delta P|}{0.5 \rho U^{2}}=0.015$ (Fig. 7a-iii), respectively; (xiii)-(xvi) symmetric Y model with hypoplastic LPA and (xiii)-(xiv) prescribed flow splits (Fig. 8a-i,ii), and (xv)-(xvi) lumped parameter models coupled at the branch outlets (Fig. $8 a$-iii,iv). Steady Newtonian flow, $R e=650$. 
Table 2: Pressure ratios with standard deviation from the mean value, as calculated from measurements of relative pressure at different points in the pulmonary branches (locations of measurement are shown at inset of Fig. 11b), under steady flow.

\begin{tabular}{|c|c|c|c|c|}
\hline & & $P_{L P A}: P_{R P A}$ & $\mathrm{P}_{\mathrm{LPA}}: \mathrm{P}_{\mathrm{MPA}}$ & $\mathrm{P}_{\mathrm{RPA}}: \mathrm{P}_{\mathrm{MPA}}$ \\
\hline (i) & T-Junction (Fig. 2 i) & $1.000 \pm 5.5 \mathrm{e}-6$ & $1.018 \pm 3.6 \mathrm{e}-3$ & $1.018 \pm 3.6 \mathrm{e}-3$ \\
\hline (ii) & $\begin{array}{l}\text { Asymmetric Y-Junction with branch origin at } \mathrm{O} \\
\text { (Fig. } 2 \text { ii) }\end{array}$ & $1.122 \pm 9.2 \mathrm{e}-3$ & $1.049 \pm 6.8 \mathrm{e}-4$ & $0.935 \pm 7.1 \mathrm{e}-3$ \\
\hline (iii) & $\begin{array}{l}\text { Asymmetric Y-Junction with displaced LPA origin } \\
\text { (Fig. } 2 \text { iii) }\end{array}$ & $1.075 \pm 7.0 \mathrm{e}-3$ & $1.043 \pm 7.1 \mathrm{e}-4$ & $0.970 \pm 6.5 \mathrm{e}-3$ \\
\hline (iv) & $\begin{array}{l}\text { Asymmetric Y-Junction with both origins displaced } \\
\text { (Fig. } 2 \text { iv) }\end{array}$ & $1.026 \pm 2.6 \mathrm{e}-3$ & $1.031 \pm 4.6 \mathrm{e}-3$ & $1.000 \pm 3.9 \mathrm{e}-3$ \\
\hline (v) & Asymmetric Y-Junction with hypoplastic LPA (Fig. 2 v) & $1.228 \pm 2.4 \mathrm{e}-2$ & $1.013 \pm 8.2 \mathrm{e}-3$ & $0.885 \pm 5.5 \mathrm{e}-3$ \\
\hline (vi) & Symmetric Y-Junction (Fig. 2 vi) & $1.000 \pm 1.6 \mathrm{e}-5$ & $1.013 \pm 3.7 \mathrm{e}-3$ & $1.013 \pm 3.6 \mathrm{e}-3$ \\
\hline (vii) & $\begin{array}{l}\text { Symmetric Y-Junction with local asymmetric stenosis in } \\
\text { LPA (Fig. } 2 \text { vii) }\end{array}$ & $0.797 \pm 1.5 \mathrm{e}-3$ & $0.793 \pm 4.8 \mathrm{e}-3$ & $0.995 \pm 4.7 \mathrm{e}-3$ \\
\hline (viii) & $\begin{array}{l}\text { Symmetric Y-Junction with local symmetric stenosis in } \\
\text { LPA (Fig. } 2 \text { viii) }\end{array}$ & $0.850 \pm 3.3 e-3$ & $0.868 \pm 2.1 \mathrm{e}-3$ & $1.021 \pm 2.6 \mathrm{e}-3$ \\
\hline (ix) & Symmetric Y-Junction hypoplastic LPA (Fig. 2 ix) & $1.145 \pm 1.6 \mathrm{e}-2$ & $1.013 \pm 7.5 \mathrm{e}-3$ & $0.885 \pm 5.5 \mathrm{e}-3$ \\
\hline (x) & Symmetric Y-Junction with extended RPA (Fig. 7a-i) & $0.937 \pm 4.3 \mathrm{e}-3$ & $0.960 \pm 5.3 \mathrm{e}-3$ & $1.024 \pm 9.5 \mathrm{e}-4$ \\
\hline (xi) & Symmetric Y-Junction with $\frac{|\Delta P|}{0.5 \rho U^{2}}=0.026$ (Fig. 7a-ii) & $1.193 \pm 1.6 \mathrm{e}-2$ & $1.085 \pm 4.6 \mathrm{e}-2$ & $0.910 \pm 3.1 \mathrm{e}-2$ \\
\hline (xii) & Symmetric Y-Junction with $\frac{|\Delta P|}{0.5 \rho U^{2}}=0.015$ (Fig. 7a-iii) & $1.071 \pm 5.6 \mathrm{e}-3$ & $1.039 \pm 2.6 \mathrm{e}-3$ & $0.970 \pm 7.5 \mathrm{e}-3$ \\
\hline (xiii) & Murray’s Law (Fig. 8a-i) & $1.150 \pm 1.5 \mathrm{e}-2$ & $1.014 \pm 7.3 \mathrm{e}-3$ & $0.882 \pm 5.6 \mathrm{e}-3$ \\
\hline (xiv) & Outflow Splitting Method (Fig. 8a-ii) & $1.097 \pm 2.1 \mathrm{e}-2$ & $1.000 \pm 1.2 \mathrm{e}-1$ & $0.910 \pm 6.3 \mathrm{e}-3$ \\
\hline$(\mathrm{xv})$ & Peripheral Resistance (Fig. 8a-iii) & $1.056 \pm 1.4 \mathrm{e}-2$ & $1.008 \pm 7.1 \mathrm{e}-3$ & $0.954 \pm 5.7 \mathrm{e}-3$ \\
\hline (xvi) & Windkessel model (Fig. 8a-iv) & $1.115 \pm 1.4 \mathrm{e}-2$ & $1.010 \pm 7.1 \mathrm{e}-3$ & $0.906 \pm 4.9 \mathrm{e}-3$ \\
\hline
\end{tabular}

Following the analysis in the pressure ratios and the identification of the stagnation point (Fig. $3,7 \mathrm{a}, 8 \mathrm{a})$, it can be observed that a $\mathrm{P}_{\mathrm{LPA}}: \mathrm{P}_{\mathrm{RPA}}$ ratio in the range of $1 \pm 0.026$ exists in the models where the stagnation point was found along the MPA centerline; that is, the T- and symmetric Y-Junctions, and the asymmetric Y-Junction with both branch origins displaced. Therefore, these models have approximately equal mean pressures in the pulmonary branches. For the remaining models, where the 
stagnation point was shifted, the $\mathrm{P}_{\mathrm{LPA}}: \mathrm{P}_{\mathrm{RPA}}$ ratio deviated further from the value of 1 , indicating the existence of differential pressures between the two branches.

In short, this study demonstrates a relation between the stagnation point and pressure ratios in the pulmonary bifurcation. These ratios also clarify that the pressure in the left pulmonary artery is generally higher than the pressure in the right and main pulmonary arteries, unless there is a local obstruction in the LPA branch and with the exception of the symmetric T- and Y-Junctions. These findings correlate well with clinical observations in TOF patients [49] indicating that measurement of the mean pressure ratios could be a useful haemodynamic index in the clinical practice for the assessment of LPA stenosis and PVR.

\subsubsection{Flow Splits}

Further to identifying pressure differences between the left and right pulmonary branches, it is also clinically relevant to estimate the branch flow splits. The flow split difference between the arterial branches becomes particularly important when taking into account the pulmonary vascular resistance. Chronic thromboembolic pulmonary hypertension (CTEPH), a type of pulmonary hypertension, develops in cases of stenosed pulmonary arterial vessels. Rarefaction, the process where the density of small capillaries and arterioles are reduced, results in elevated pulmonary vascular resistance, and flow in this case is diverted towards the non-stenosed branch [50, 51]. Furthermore, long-term pulmonary stenosis in patients with TOF is thought to lead to differential lung growth [52]. Many studies have focused their investigations towards the effect of pulmonary stenosis and hypertension on the distribution of flow between the left and right pulmonary arteries. Spazzapan et al. (2018) [51] investigated the flow split in stenosed arteries of patients with CTEPH and found that the percentage of flow directed towards the stenosed branch was between $22 \%$ to $46 \%$, depending on the degree of stenosis. An increase of $10 \%-60 \%$ in the flow directed towards the previously stenosed branch is reported upon removal of stenosis [51]. Pekkan et al. (2005) [53] investigated the effect of left pulmonary stenosis in patients with total cavopulmonary connection, showing that decreased stenosis resulted in a more balanced flow split in the pulmonary arteries which improved lung perfusion. Pressure values in their models, with reference to the LPA pressure, were also found to increase in hypoplastic branches, more than the local stenosis. They concluded that a uniform calibre (also called diffuse stenosis) results in reduced left lung perfusion. In another study of Schiavazzi et al. (2015) [54], the effect of pulmonary stenosis was also considered in single-ventricle patients. Their results indicated that the flow split between the LPA and RPA becomes clinically important when local stenosis is greater than $65 \%$, where the percentage of flow to the LPA is less than $30 \%$ and the pressure drop in the LPA is higher than $3.0 \mathrm{mmHg}$.

Nevertheless, it is still ambiguous whether surgical repair of the pulmonary stenosis will benefit the patient. In the study of Cheng et al. (2005) [55], computed flow splits in the RPA and LPA branches of healthy children were compared with those from the study of Pederson et al. (2002) [56] from children with cavopulmonary connection, and the ratios were similar. In addition, Spilker et al. (2007) [57] found no apparent benefit to a patient with surgically repaired stenosis. Furthermore, Kheyfets et al. (2015) [58], investigated the flow in the pulmonary arterial network of a healthy patient using various outflow boundary conditions (zero-traction, a constant peripheral resistance, and an arterial tree of several generations) and found minimal impact on the proximal arteries but considerable effect on the flow distribution in distal tree vessels.

The above studies demonstrate that peripheral pulmonary vascular resistance and downstream pressure may influence the flow rates within the pulmonary arteries. Clinical measurement of the flow splits between the LPA and RPA branches may therefore indicate altered peripheral and proximal conditions, and serve as another haemodynamic marker for PVR evaluation.

In the results presented here (Fig. 7, 8), peripheral resistance and downstream pressure altered the flow splits in the daughter branches. Table 3 summarises different flow split percentages for nine of the cases presented, with flow splits other than 50:50\%, as calculated based on velocity profiles at a distance of $3 \mathrm{~cm}(1.15 \mathrm{D})$ from the branch origin for each model. The symmetric and asymmetric hypoplastic models significantly reduce the flow rate in the left branch; a flow split of approximately $12: 88 \%\left(\mathrm{Q}_{\mathrm{LPA}}: \mathrm{Q}_{\mathrm{RPA}}\right)$ was observed, which is considered an extreme case [54]. Nevertheless, similar 
flow ratios were predicted with Murray's law $\left(11: 89 \% \mathrm{Q}_{\mathrm{LPA}}: \mathrm{Q}_{\mathrm{RPA}}\right)$, and with a three-element Windkessel model (14:86\% Q Q "outflow splitting" method [35] (20:80\% $\left.\mathrm{Q}_{\mathrm{LPA}}: \mathrm{Q}_{\mathrm{RPA}}\right)$, and the coupling of a peripheral resistance $\left(22: 78 \% \mathrm{Q}_{\mathrm{LPA}}: \mathrm{Q}_{\mathrm{RPA}}\right)$, accounting to a maximum of $10 \%$ difference compared to a zero pressure boundary (Fig. 8a, 9a). In the study of Vignon-Clementel et al., (2006) [27], a 20\% difference in the flow splits (50:50\% vs. 30:70\%) was found when using, respectively, an impedance boundary condition and a constant pressure $(90 \mathrm{mmHg})$, in a 3D symmetric stenotic model with a $75 \%$ reduction in the area within the stenosis and pulsatile inflow. This deviation might be explained due to the dissimilarity between the two geometries. More physiologically realistic flow splits of 33:67\% and 40:60\% $\left(\mathrm{Q}_{\mathrm{LPA}}: \mathrm{Q}_{\mathrm{RPA}}\right)$, were achieved by modifying the length or the outlet pressure of the branch (Table 3 ), representing different peripheral pressure conditions. Similar values, in the range of 40:60\% to 51:49\% $\left(Q_{L P A}: Q_{R P A}\right)$ have been observed in healthy subjects [34]. Patients with repaired tetralogy of Fallot may exhibit flow ratios within the normal range of 39:61 to 57:43 (Q $\mathrm{Q}_{\mathrm{LPA}}: \mathrm{Q}_{\mathrm{RPA}}$ ) [11]. Abnormally low flow rates have been also reported in the literature for congenital heart patients, with flow percentages less than $39 \%$ to the left, or less than $43 \%$ to the right lung [11,59].

Overall, a correlation appears to exist between the WSS distribution, the pressure ratios and the flow splits in the bifurcating models analysed in this study. More specifically, in the presence of different flow splits between the daughter branches (Table 3), the peak WSS value (Fig. 7c, 8b) and the pressure (Table 2) decreased in the branch with the higher flow rate; for example, cases Fig. 7a-i and 7a-ii. The only exception in this trend is for cases with a flow split greater than $12: 88 \%\left(\mathrm{Q}_{\mathrm{LPA}}: \mathrm{Q}_{\mathrm{RPA}}\right)$ where the peak WSS value was slightly higher in the RPA (Fig. 6d and Fig 8a i).

Table 3: Flow Split percentages at the left and the right pulmonary branches, as calculated using velocity profiles data, extracted at a distance of $3 \mathrm{~cm}(1.15 \mathrm{D})$ from the branch origin of each model.

\begin{tabular}{|c|c|c|c|c|c|}
\hline \multicolumn{2}{|c|}{ Type of Y-Junction } & \multirow{2}{*}{$\begin{array}{c}\begin{array}{c}\text { Branch } \\
\text { diameter }\end{array} \\
\left(\mathrm{D}_{\mathrm{LPA}}: \mathrm{D}_{\mathrm{RPA}}\right) \\
1: 2\end{array}$} & \multirow{2}{*}{ 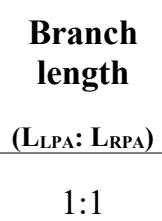 } & \multirow{2}{*}{$\begin{array}{c}\frac{|\Delta P|}{0.5 \rho U^{2}} \\
0\end{array}$} & \multirow{2}{*}{$\begin{array}{c}\begin{array}{c}\text { Flow Split } \\
\text { (\%) }\end{array} \\
\left(\sim Q_{\mathrm{LPA}}: \sim \mathbf{Q}_{\mathrm{RPA}}\right) \\
12: 88\end{array}$} \\
\hline Asymmetric & with hypoplastic LPA & & & & \\
\hline \multirow{8}{*}{ Symmetric } & with hypoplastic LPA & $1: 2$ & $1: 1$ & 0 & $12: 88$ \\
\hline & With extended RPA & $1: 1$ & $1: 2$ & 0 & $67: 33$ \\
\hline & with $\frac{|\Delta P|}{0.5 \rho U^{2}}=0.026$ & $1: 1$ & $1: 1$ & 0.026 & $33: 67$ \\
\hline & $\frac{\text { with }|\Delta P|}{0.5 \rho U^{2}}=0.015$ & $1: 1$ & $1: 1$ & 0.015 & $40: 60$ \\
\hline & $\begin{array}{l}\text { with hypoplastic LPA - } \\
\text { Murray's Law }\end{array}$ & \multirow{4}{*}{$1: 2$} & \multirow{4}{*}{$1: 1$} & \multirow{4}{*}{0} & 11:89 \\
\hline & $\begin{array}{l}\text { with hypoplastic LPA - } \\
\text { "outflow splitting method" }\end{array}$ & & & & $20: 80$ \\
\hline & $\begin{array}{l}\text { with hypoplastic LPA - } \\
\text { peripheral resistance }\end{array}$ & & & & $22: 78$ \\
\hline & $\begin{array}{l}\text { with hypoplastic LPA - } \\
\text { Windkessel model }\end{array}$ & & & & $14: 86$ \\
\hline
\end{tabular}

\section{Concluding remarks}

Congenital heart defects represent a great challenge in the medical sector and patients with tetralogy of Fallot are commonly diagnosed with pulmonary valve regurgitation and pulmonary local 
stenosis, hypoplastic LPA, or with LPA kinking. These complications are associated with RV dysfunction, and pulmonary valve replacement is deemed necessary.

Despite some simplifications, overall, this study highlights some previously undocumented aspects of the flow in bifurcating geometries, such as the pulmonary bifurcation: The geometric location of the flow divider, which depends on the geometry and the branch origin, influences the WSS distribution along the inner wall of the bifurcation (Fig. 6b, 7c, 8b). A stagnation point that is shifted from the MPA centreline encourages differential pressures $\left(\mathrm{P}_{\mathrm{LPA}}: \mathrm{P}_{\mathrm{RPA}} \neq 1\right)$ in geometries with the same downstream pressure conditions (Table 2 (i)-(x)), and, vice versa, altered peripheral conditions affect the area of flow impingement (Fig. 7a-ii,iii, 8a). Our results also clarify that increased recirculation is not only an effect of increased branching angle, as suggested in previous studies [3], but also of the origin of the pulmonary branches (Fig. 5). More importantly, this work explains that the pressure in the left pulmonary artery is generally more elevated compared to the pressure in the right and main pulmonary arteries, unless there is a local obstruction in the LPA branch and with the exception of the symmetric T- and Y- Junctions (Fig. 9b). In addition, it demonstrates that downstream pressure conditions and peripheral resistance alter the flow in the pulmonary arteries and explain the occurrence of different flow splits between the branches. This, in turn, appears to reduce the peak WSS and pressure in the high flow-rate branch, for flows up to $12: 88 \%$, which may help assess pulmonary branch regurgitations. Finally, this work clarified some aspects of clinical observations in TOF patients through measurements of the mean pressure ratios in the pulmonary bifurcation, a haemodynamic index which could potentially contribute to the assessment of LPA stenosis, RV dysfunction, and PVR.

The interrelation among the right ventricle, pulmonary valve and flow in anatomically varying pulmonary arteries is very complex and further studies in anatomically-realistic models are necessary. Future work will involve the reconstruction of three-dimensional patient-specific models using MRI or CT data, of adult patients with repaired tetralogy of Fallot, and the assignment of more realistic boundary conditions. Patient-specific data can be incorporated in the models, the importance of which is extensively discussed in the literature [31, 60].

Competing interests: None declared

Funding: This work was supported in part from the University of Strathclyde Research Studentship Scheme (SRSS) Research Excellence Awards (REA), Project No 1208, and from the European Union's Horizon 2020 research and innovation programme under the Marie Skłodowska-Curie grant agreement No 749185 .

Ethical approval: Fig. 2a was obtained with ethical approval from the Glasgow CRIF approval group, and written informed consent (REC name WoS REC3, reference 16/WS/0220).

Acknowledgements: The authors would like to thank Dr Pauline Hall-Barientos for providing the MRI image (Fig. 2a) of a pulmonary bifurcation from a healthy volunteer. Parts of the results were obtained using the ARCHIE-WeSt High Performance Computer (www.archie-west.ac.uk) based at the University of Strathclyde.

* Corresponding author: Department of Biomedical Engineering, University of Strathclyde, 106 Rottenrow East, Glasgow G4 0NW. Tel: 0141548 3228. Email: asimina.kazakidi@strath.ac.uk

\section{References}

[1] S.C. Mitchell, S.B. Korones, H.W. Berendes, Congenital Heart Disease in 56,109 Births Incidence and Natural History. Circulation (43) (1971) 323-332.

[2] B.N.S. Rao, R.C. Anderson, J.E. Edwards, Anatomic variations in the tetralogy of Fallot. American Heart Journal (81) (1971) 361-371.

[3] W. Zhang, J. Liu, Q. Yan, J. Liu, H. Hong, L. Mao, Computational haemodynamic analysis of left pulmonary artery angulation effects on pulmonary blood flow. Interactive CardioVascular and Thoracic Surgery (23) (2016) 519-525.

[4] S. Babu-Narayan, M.A. Gatzoulis, Tetralogy of Fallot. In: Diagnosis and Management of Adult 
Congenital Heart Disease. (2018) 474-488. Elsevier.

[5] M.A. Harris, K.K. Whitehead, M.J. Gillespie, T.Y. Liu, M.T. Cosulich, D.C. Shin, E. Goldmuntz, P.M. Weinberg, M.A. Fogel, Differential Branch Pulmonary Artery Regurgitant Fraction is a Function of Differential Pulmonary Arterial Anatomy and Pulmonary Vascular Resistance. Cardiovascular Imaging (4) (2011) 506-513.

[6] T. Geva, Repaired tetralogy of Fallot: the roles of cardiovascular magnetic resonance in evaluating pathophysiology and for pulmonary valve replacement decision support. Journal of Cardiovascular Magnetic Resonance (13) (2011) 1-24.

[7] F.J. Puga, D.C. McGoon, P.R. Julsrud, G.K. Danielson, D.D Mail, Complete Repair of Pulmonary Atresia with Nonconfluent Pulmonary Arteries. The Annals of Thoracic Surgery (35) (1982) 36-44.

[8] R.R. Chaturvedi, P.J. Kilner, P.A. White, A. Bishop, R. Szwarc, A.N. Redington, Increased Airway Pressure and Simulated Branch Pulmonary Artery Stenosis Increase Pulmonary Regurgitation After Repair of Tetralogy of Fallot. Circulation (95) (1997) 643-649.

[9] D.B. McElhinney, A.J. Parry, M. Reddy, F.L. Hanley, P. Stanger, Left Pulmonary Artery Kinking Caused by Outflow Tract Dilation After Transannular Patch Repair of Tetralogy of Fallot. The Annals of Thoracic Surgery (65) (1998) 1120-1126.

[10] M. Kordybach-Prokopiuk., M. Kowalski, M. Spiewak, E. Piotrowicz, P. Hoffman, What can pulmonary regurgitation indices reflect in patients after tetralogy of Fallot repair?. Kardiologia Polska (1) (2015) 31-39.

[11] I.S. Kang, A.N. Redington, L.N. Benson, C. Macgowan, E.R. Valsangiacomo, K. Roman, C. Kellenberger, S.J. Yoo, Differential regurgitation in branch pulmonary arteries after repair of tetralogy of Fallot. Circulation (107) (2003) 2938-2943.

[12] D.A. Rubenstein, W. Yin, M.D. Frame, Biofluid Mechanics (Second Edition). Academic Press (2015) 63-223.

[13] C.D. Murray, The physiological principle of minimum work applied to the angle of branching of arteries. The Journal of General Physiology (9) (1926) 835-841.

[14] T.J. Pedley, R.C. Schroter, M.F. Sudlow, Flow and pressure drop in systems of repeatedly branching tubes. Journal of Fluid Mechanics (46) (1971) 365-383.

[15] T. Sochi, Fluid Flow at Branching Junctions. International Journal of Fluid Mechanics Research (42) (2015) 59-81.

[16] F. Capuano, Y.-H. Loke, E. Balaras, Blood Flow Dynamics at the Pulmonary Bifurcation. Fluids (4) (2019) 1-20.

[17] M.M. Samyn, J.F. LaDisa, Novel Applications of Cardiovascular Magnetic Resonance ImagingBased Computational Fluid Dynamics Modeling in Pediatric Cardiovascular and Congenital Heart Disease. In: Assessment of Cellular and Organ Function and Dysfunction using Direct and Derived MRI Methodologies (2016) 27-56, InTech.

[18] R. Guibert, K. McLeod, A. Caiazzo, T. Mansi, M.A. Fernandez, M. Sermesant, X. Pennec, I. Vignon-Clementel, Y. Boudjemline, J.F. Gerbeau, Group-wise construction of reduced models for understanding and characterization of pulmonary blood flows from medical images. Medical Image Analysis (18) (2014) 63-82.

[19] M.J. Chern, M.T. Wu, H.L. Wang, Numerical investigation of regurgitation phenomena in pulmonary arteries of Tetralogy of Fallot patients after repair. Journal of Biomechanics (41) (2008) 3002-3009.

[20] M.J. Chern, M.T. Wu, S. Wei-Her, Numerical Study for Blood Flow in Pulmonary Arteries after Repair of Tetralogy of Fallot. Computational and Mathematical Methods in Medicine (2012) (2012)1- 
18.

[21] C. Alegre-Martínez, K.-S. Choi, O. Tammisola, D. McNally, On the axial distribution of plaque stress: Influence of stenosis severity, lipid core stiffness, lipid core length and fibrous cap steffness. Medical Engineering and Physics (68) (2019) 76-84.

[22] B.T. Tang, S.S. Pickard, F.P. Chan, P.S. Tsao, C.A. Taylor, J.A. Feinstein, Wall shear stress is decreased in the pulmonary arteries of patients with pulmonary arterial hypertension: An image-based, computational fluid dynamics study. Pulmonary Circulation, (2) (2012) 470-476.

[23] V.J.S. Nina, Surgical Repair of Stenotic Pulmonary Arteries in Tetralogy of Fallot. In: Cardiac Surgery-A Commitment to Science, Technology and Creativity (Maluf M.A, Evora R.B, ed), (2014) 125-147. IntechOpen.

[24] W.S Jang, W.-H. Kim, S. Cho, Effects of Angle Correction Angioplasty for Pulmonary Artery Stenosis With Tetralogy of Fallot. Ann Thorac Surg (103) (2017) 862-868.

[25] T. Defraeye, B. Blocken, J. Carmeliet, CFD analysis of convective heat transfer at the surfaces of a cube immersed in a turbulent boundary layer. International Journal of Heat and Mass Transfer (53) (2010) 297-308.

[26] M. Boumpouli, M. Danton, T. Gourlay, M. Kazakidi, Hemodynamics in the Pulmonary Bifurcation in Relation to Adults with Congenital Heart Disease: Effect of Branching Angle and Origin. $6^{\text {th }}$ European Conference on Computational Mechanics- $7^{\text {th }}$ European Conference on Computational Fluid Dynamics (2018) 1-12.

[27] I.E. Vignon-Clementel, C.A. Figueroa, K.E., Jansen, C.A. Taylor, Outflow boundary conditions for three-dimensional finite element modeling of blood flow and pressure in arteries. Comput. Methods Appl. Mech. Engrg. (195) (2006) 3776-3796.

[28] I.T. Gabe, J.H. Gault, J. Ross, D.T. Mason, C.J. Mills, J.P. Schillingford, E. Braunwald, Measurements of instantaneous blood flow velocity and pressure in conscious man with a catheter-tip velocity probe. Circulation (40) (1969) 603-614.

[29] J. Bronzino, In: The Biomedical Engineering Handbook, Second Edition. CRC Press LLC, (2000) 30-33.

[30] P. Neofytou, C. Housiadas, S.G. Tsangaris, A.K. Stubos, D.I. Fotiadis, Newtonian and Power-Law fluid flow in a T-Junction of rectangular ducts. Theoretical and Computational Fluid Dynamics (28) (2014) 233-256.

[31] A. Young, Patient-Specific Mathematical Modelling of the Hybrid Procedure in the Treatment of Hypoplastic Left Heart Syndrome, PhD thesis, University of Strathclyde, Glasgow (2014).

[32] M. McElroy, A. Keshmiri, Impact of Using Conventional Inlet/Outlet Boundary Conditions on Haemodynamic Metrics in a Subject-Specific Rabbit Aorta. Journal of Engineering in Medicine (232) (2018) 103-113.

[33] M.U. Qureshi, N.A. Hill, A computational study of pressure wave reflections in the pulmonary arteries. Journal of Mathematical Biology (71) (2015) 1525-1549.

[34] B.T. Tang, T.A. Fonte, F.R. Chan, P.S. Tsao, J.A. Feinstein, C.A. Taylor, Three-dimensional hemodynamics in the human pulmonary arteries under resting and exercise conditions, (39) (2010) 347 358.

[35] C. Chnafa, O. Brina, V.M. Pereira, D.A. Steinman, Better Than Nothing: A Rational Approach for Minimizing the Impact of Outflow Strategy on Cerebrovascular Simulations. American Journal of Neuroradiology (39) (2018) 337-343.

[36] L. Grinberg, G.E. Karniadakis, Outflow Boundary Conditions for Arterial Networks with Multiple Outlets. Annals of Biomedical Engineering (36) (2008) 1496-1514. 
[37] D. Katritsis, L. Kaiktsis, A. Chaniotis, J. Pantos, E.P. Efstathopoulos, V. Marmarelis, Wall shear stress: Theoretical considerations and methods of measurement. Progress in Cardiovascular Disease, (49) (2007) 307-329.

[38] D. Liepsch, S. Moravec, Measurement and calculations of laminar flow in a ninety degree bifurcation. Journal of Biomechanics (15) (1982) 473-485.

[39] A. Kazakidi, S.J. Sherwin, P.D. Weinberg, Effect of Reynolds number and flow division on patterns of haemodynamic wall shear stress near branch points in the descending thoracic aorta. Journal of the Royal Society Interface (6) (2009) 539-548.

[40] A. Kazakidi, A.M. Plata, S.J. Sherwin, P.D. Weinberg, Effect of reverse flow on the pattern of wall shear stress near arterial branches. Journal of the Royal Society Interface (8) (2011) 1594-1603.

[41] T. Thenappan, K.W. Prins, M.R. Pritzker, J. Scandurra, K. Volmers, E.K. Weir, The critical role of pulmonary arterial compliance in pulmonary hypertension. Annals of the American Thoracic Society, (13) (2016) 276-284.

[42] K. Kanokjaruvijit, T. Donprai-on, N. Phanthura, P. Noidet, J. Siripokharattana, Wall shear stress and velocity distributions in different types of stenotic bifurcations. Journal of Mechanical Science and Technology (31) (2017) 2339-2349.

[43] D.G. Katritsis, A. Theodorakakos, I. Pantos, A. Andriotis, E.P. Efstathopoulos, G. Siontis, N. Karcanias, S. Redwood, M. Gavaises, Vortex formation and recirculation zones in left anterior descending artery stenoses: computational fluid dynamics analysis. Physics in Medicine and Biology (55) (2010) 1395-1411.

[44] M.P. Szymanski, E. Metaxa, H. Meng, J. Kolega, Endothelial Cell Layer Subjected to Impinging Flow Mimicking the Apex of an Arterial Bifurcation. Annals of Biomedical Engineering (36) (2008) 1681-1689.

[45] S.S.M. Chen, P.J. Kilner, Unilateral pulmonary artery branch stenosis: Diastolic prolongation of forward flow appears to maintain flow to the affected lung if the pulmonary valve is competent. International Journal of Cardiology (168) (2013) 3698-3703.

[46] A. Young, T. Gourlay, S. McKee, M. H.D. Danton, Computational modelling of the hybrid procedure in hypoplastic left heart syndrome: A comparison of zero-dimensional and three-dimensional approach. Medical Engineering and Physics (36) (2014) 1549-1553.

[47] N.H.J. Pijls, J.A.M. van Son, R.L. Kirkeeide, B. De Bruyne, K.L. Gould, Experimental basis of determining maximum coronary, myocardial, and collateral blood flow by pressure measurements for assessing functional stenosis severity before and after percutaneous transluminal coronary angioplasty. Circulation (86) (1993) 1354-1367.

[48] G. Arbia, C. Corsini, M.E. Moghadam, A.L. Marsden, F. Migliavacca, G. Pennati, T.Y. Hsia, I.E. Vignon-Clementel, Numerical blood flow simulation in surgical corrections: what do we need for an accurate analysis? Journal of Surgical Research, (186) (2014) 44-55.

[49] A.J. Muster, A. Van Grondelle, M.H. Paul, Unequal Pressures in the central pulmonary arterial branches in patients with pulmonary stenosis. Pediatric Cardiology (2) (1982) 7-14.

[50] M.S. Olufsen, N.A. Hill, G.D.A. Vaughan, C. Sainsbury, M. Johnson, Rarefaction and blood pressure in systemic and pulmonary arteries. Journal of Fluid Mechanics, (705) (2012) 280-305.

[51] M. Spazzapan, P. Sastry, J. Dunning, D. Nordsletten, A. de Vecchi, The use of Biophysical Flow Models in the Surgical Management of Patients Affected by Chronic Thromboembolic Pulmonary Hypertension. Frontiers in Physiology, (9) (2018) 1-13.

[52] M.N. Ilbawi, F.S. Idriss, S.Y. DeLeon, A.J. Muster, S.S Gidding, T.E. Berry, M.H, Paul, Factors that exaggerate the deleterious effects of pulmonary insufficiency on the right ventricle after tetralogy 
repair. The Journal of Thoracic and Cardiovascular Surgery, (93) (1987) 36-44.

[53] K. Pekkan, H. D. Kitajima, D. de Zelicourt, J.M. Forbess, W.J. Parks, M.A. Fogel, S. Sharma, K.R. Kanter, D. Frakes, A.P. Yoganathan, Total cavopulmonary connection flow with functional left pulmonary artery stenosis: angioplasty and fenestration in vitro. Journal of the Americal Heart Association, (112) (2005) 3264-3271.

[54] D.E. Schiavazzi, E.O. Kung, A.L. Marsden, C. Baker, G. Pennati, T.Y. Hsia, A. Hlavacek, A.L. Dorfman, Hemodynamic effects of left pulmonary artery stenosis after superior cavopulmonary connection: A patient-specific multiscale modeling study. The Journal of Thoracic and Cardiovascular Surgery, (149) (2015) 689-696.[43] P. Bachler, N. Pinochet, J. Sotelo, G. Crelier, P. Irarrazaval, C. Tejos, S. Uribe, Assessment of normal flow patterns in the pulmonary circulation by using 4D magnetic resonance mapping. Magnetic Resonance Imaging (31) (2013) 178-188.

[55] C.P Cheng, R.J. Herfkens, C.A. Taylor, J.A. Feinstein, Proximal pulmonary artery blood flow characteristics in healthy subjects measured in an upright posture using MRI: The Effects of Exercise and Age. Journal of Magnetic Resonance Imaging, (21) (2005) 752-758.

[56] E.M, Pederson, E.V Stenbog, T. Frund, Flow during exercise in the total cavopulmonary connection measured by magnetic resonance velocity mapping. Heart, (87) (2002) 554-558.

[57] R.L. Spilker, J.A. Feinstein, D.W. Parker, V.M. Reddy, C.A. Taylor, Morphometry-based impedance boundary conditions for patient-specific modeling of blood flow in pulmonary arteries. Annals of Biomedical Engineering, (35) (2007) 546-559.

[58] V.O. Kheyfets, L. Rios, T. Smith, T. Schroeder, J. Mueller, S. Murali, D. Lasorda, A. Zikos, J. Spotti, J.J. Reilly Jr, E.A. Finol, Patient-specific computational modeling of blood flow in the pulmonary arterial circulation. Computer Methods and Programs in Biomedicine, (120) (2015) 88-101.

[59] P. Bachler, N. Pinochet, J. Sotelo, G. Crelier, P. Irarrazaval, C. Tejos, S. Uribe, Assessment of normal flow patterns in the pulmonary circulation by using 4D magnetic resonance mapping. Magnetic Resonance Imaging (31) (2013) 178-188.

[60] M.A. Van Doormaal, A. Kazakidi, M. Wylezinska, A. Hunt, J.L. Tremoleda, A. Prottis, Y. Bohraus, W. Gsell, P.D. Weinberg, C.R. Ethier, Haemodynamics in the mouse aortic arch computed from MRI-derived velocities at the aortic root. Journal of the Royal Society Interface (9) (2012) 28342844. 


\section{Supplementary Methods}

A sensitivity analysis was performed in the symmetric Y-Junction model to investigate the effects of various different boundary conditions on the original solution, as follows:

Tapering in the bifurcation: Tapering is an unequivocal characteristic of arterial conduits, from the larger arteries to the smaller ones, based on multiple studies [1,2]. To investigate this effect a geometry was created, with the proximal main pulmonary artery (MPA), left pulmonary artery (LPA), and right pulmonary artery (RPA) RPA diameters of the tapered model kept equal to the non-tapered model, and the distal diameters modified to $\sim 0.95 D$ for the MPA and $\sim 0.75 \mathrm{D}$ for both daughter branches; $D$ is the diameter of the main pulmonary artery (MPA) assumed $2.6 \mathrm{~cm}$. These reductions in diameters correspond to a $4 \%$ and a $2 \%$ tapering, respectively, which are representative of the percentages found in a healthy young male [2]. No difference was found for the integral of the streamwise velocity profile between the non-tapered and tapered asymmetric models, as measured at the entrance of the bifurcation (at a distance $0.01 \mathrm{~m}$ from the origin $\mathrm{O}$ ).

An additional three-dimensional (3D) symmetric Y-Junction geometry was created based on the two-dimensional tapered model. The computational mesh of the 3D geometry consisted primarily of prismatic elements, with tetrahedral elements near the walls. A boundary layer was added near the arterial wall boundaries to capture the viscous effects, which included 17 layers with the first layer being at a distance of $\sim 0.002 D$ away from the wall. The total number of elements was $\sim 3800000$.

Velocity profile at the inlet and MPA length: The sensitivity of the steady velocity profile at the inlet of the MPA was investigated, first, through the use of a uniform inlet profile to represent blood flow emanating from the pulmonary valve, and, second, by reducing the length of the MPA to $\sim 2 D$ (equal to $5 \mathrm{~cm}$ ), in order to reflect a more physiological MPA dimension [2]. The uniform velocity profile was assumed with the same mean $U$ value as the parabolic profile.

Time-dependent flow: To account for the unsteady nature of blood flow, a sinusoidal variation of the velocity profile at the MPA inlet was assumed, based on the following expression:

$$
u(x, t)=u_{\text {par }}[1+\sin (2 \pi f t)]
$$

where $u_{\text {par }}(x)$ is given by Eq. (2),

$$
u_{p a r}(x)=\frac{3}{2} U\left(1-\frac{4 x^{2}}{D^{2}}\right)
$$

and $f$ is the frequency, assumed as $2 \mathrm{~Hz}$ [3]. Time-averaged results over a single cycle were compared with the steady-state numerical results for the same mean velocity.

Turbulent flow model: The simulations were performed assuming the k- $\omega$ shear stress transport (SST) turbulence flow model (with model specifications as presented in Table 1) [4], calculated using the following formulas: turbulent intensity $\left(I=0.16^{*} R e_{D}{ }^{-1 / 8}\right)$, turbulent energy $\left(k=3 / 2 *(U I)^{2}\right)$, turbulent length scale $\left(L=0.038^{*} D\right)$, and specific dissipation rate $(\omega=\sqrt{k} / L)$.

Reynolds number with turbulent flow model: The effect of Reynolds number was tested by increasing the Reynolds number from 650 to 1950 and 3250, which correspond to an increase of the mean velocity $U$ from $0.1 \mathrm{~m} / \mathrm{s}$ to $0.3 \mathrm{~m} / \mathrm{s}$ and $0.5 \mathrm{~m} / \mathrm{s}$, respectively, for the specific MPA diameter, D. Such values of velocities can be observed in the pulmonary bifurcation during systole [5]. The simulations were performed using the turbulent flow model.

Table 1: Parameters used for the k- $\omega$ SST turbulent model (U: mean velocity, Re: Reynolds number, I: turbulence intensity,

\begin{tabular}{|c|c|c|c|c|c|}
\hline$U(\mathrm{~m} / \mathrm{s})$ & $\mathbf{R e}$ & I & $k\left(m^{2} / s^{2}\right)$ & $L(\mathrm{~m})$ & $\Omega(1 / s)$ \\
\hline 0.1 & 650 & 0.071 & $7.6 e-05$ & $9.88 \mathrm{e}-04$ & 8.83 \\
\hline 0.3 & 1950 & 0.062 & $5.2 \mathrm{e}-4$ & $9.88 \mathrm{e}-04$ & 23.08 \\
\hline 0.5 & 3250 & 0.058 & $1.2 \mathrm{e}-03$ & $9.88 \mathrm{e}-04$ & 36.10 \\
\hline
\end{tabular}
$k$ : turbulent energy, $l:$ turbulent length scale, and $\omega$ : specific dissipation rate). 
Inlet flow condition for the three-dimensional model: The three-dimensional symmetric Y-Junction was tested by assuming a fully developed velocity profile at the MPA inlet, for both steady and unsteady flow, with the same mean velocity $U$.

Non-Newtonian models: Additional simulations considered the non-Newtonian rheology of blood. For that, four different models were tested, namely the power law, the Cross power law, and the Casson and Bird-Carreau models, with model specifications presented in Table 2 [6]. The power law is the simplest non-Newtonian model to be considered. The Bird-Carreau and the Cross power law models have viscosities very close to the Newtonian one at high shear rates. In addition, the cross model considers a short rate of viscosities and therefore could be a good candidate for simulating blood flow in large arteries. Finally, the Casson model is expressed in terms of the hematocrit, which is assumed $40 \%$ for normal blood [6].

Table 2: Non-Newtonian models of blood flow ( $v$ : blood viscosity, $v_{\infty}$ : infinite shear rate viscosity, $v_{o}$ : zero shear rate viscosity, $k$ : relaxation time constant, $\dot{\gamma}$ : shear rate, $\alpha$ : constant parameter, $n:$ power law index, $\tau_{0}:$ yield stress). Wall shear stress values at the inlet MPA walls of the symmetric Y-Junction for the different models, are also presented.

\begin{tabular}{|c|c|c|c|}
\hline Rheological Model & Viscosity & Specified Values & WSS at inlet MPA wall (dynes $\left./ \mathrm{cm}^{2}\right)$ \\
\hline Newtonian & $v$ & $v: 0.004 \mathrm{~Pa} \mathrm{~s}$ & 0.922 \\
\hline Power Law & $v=(k \dot{\gamma})^{n-1}, v_{\min } \leq v \leq v_{\max }$ & $\begin{array}{l}\nu_{\max }: 0.056 \mathrm{~Pa} \mathrm{~s} \\
v_{\min }: 0.0035 \mathrm{~Pa} \mathrm{~s} \\
\mathrm{k}: 0.017 \mathrm{~s}, \mathrm{n}: 0.708\end{array}$ & 1.696 \\
\hline Cross power law & $v=v_{\infty}+\frac{v_{0}-v_{\infty}}{1+(m \dot{\gamma})^{\alpha}}$ & $\begin{array}{l}\nu_{\infty}: 0.0035 \mathrm{~Pa} \mathrm{~s}, \\
\nu_{o}: 0.0364 \mathrm{~Pa} \mathrm{~s}, \\
\mathrm{~m}: 0.38 \mathrm{~s} \text { (consistency } \\
\text { index), } \alpha: 1.45\end{array}$ & 1.229 \\
\hline Casson & $v=\left(\sqrt{\tau_{0} / \dot{\gamma}}+\sqrt{m}\right)^{2}$ & $\begin{array}{l}\mathrm{m}: 0.00414 \mathrm{~Pa} \mathrm{~s} \\
\text { (viscosity coefficient), } \\
\tau_{0}: 0.0038 \mathrm{~Pa}\end{array}$ & 1.198 \\
\hline Bird-Carreau model & $\begin{array}{l}v \\
=v_{\infty} \\
+\left(v_{o}-v_{\infty}\right)\left[1+(k \dot{\gamma})^{\alpha}\right]^{(n-1) / \alpha}\end{array}$ & $\begin{array}{l}v_{\infty}: 0.0035 \mathrm{~Pa} \mathrm{~s} \\
v_{0}: 0.056 \mathrm{~Pa} \mathrm{~s} \\
\mathrm{k}: 3.313 \mathrm{~s}, \mathrm{n}: 0.3568\end{array}$ & 1.696 \\
\hline
\end{tabular}

\section{Post-processing}

For the non-Newtonian model, WSS values were non-dimensionalised with values calculated at the inlet of a straight geometry with the same MPA diameter and flow conditions (last column of Table 2). The time-averaged wall shear stress was further used and was determined according to the following equation:

$$
\tau_{\text {mean }}=\frac{1}{T} \int_{0}^{T}\left|\tau_{w}\right| d t
$$

where $\left|\tau_{w}\right|$ is the magnitude of the instantaneous WSS vector.

\section{Verification and Validation}

The comparison between OpenFOAM ${ }^{\circledR}$ and ANSYS Fluent ${ }^{\circledR}$, mentioned in Section 2.7 of the manuscript, is shown in Fig. 1a for non-dimensionalised velocity contours. A percentage difference of $0.047 \%$ was quantified for the integral of velocity profiles (Fig. 1b), positioned at the entrance of the bifurcation (at a distance $0.01 \mathrm{~m}$ from the origin $\mathrm{O}$ ). 
(a)

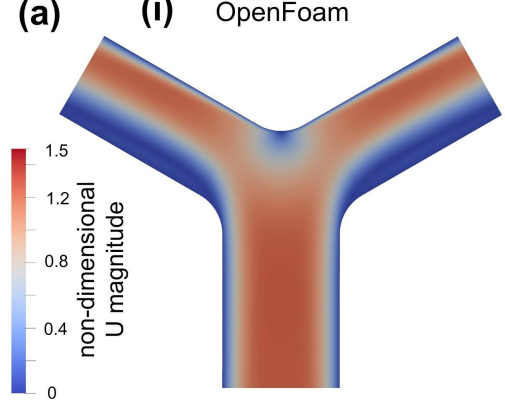

(ii)

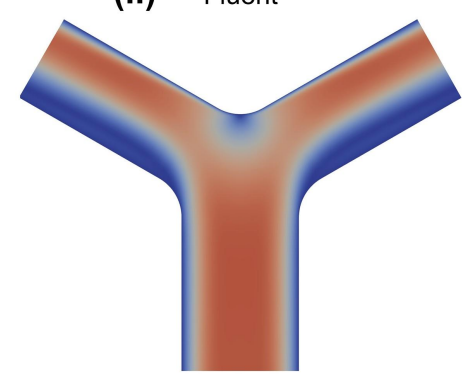

(b)

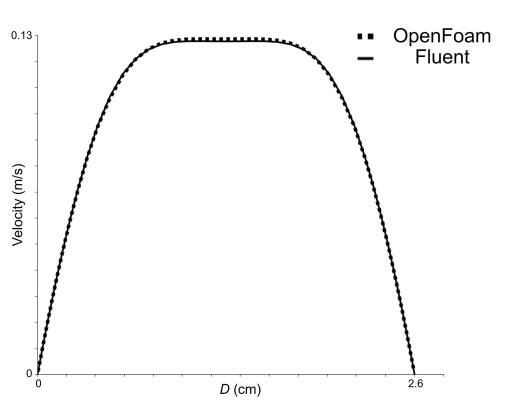

Fig. 1: Comparison of OpenFoam and Ansys Fluent. (a) Non-dimensionalised velocity contours. (b) Velocity profiles at the entrance of the bifurcation and at a distance of $0.01 \mathrm{~m}$ from the branch origin. Steady Newtonian flow, $R e=650$.

\section{Supplementary Results}

\section{Steady inlet velocity profile and MPA length}

Fig. 2a presents velocity contours overlaid by streamlines of velocity, and the insets show the respective velocity profiles, non-dimensionalised by the mean MPA velocity. The length of the MPA had no effect on the velocity distribution in the models with the same parabolic inlet profile, as expected (top row of Fig. 2a). However, there was an overall decrease in the maximum velocity values in the daughter branches, for the models with uniform inlet profiles, for both MPA lengths (bottom row of Fig. 2a), with the most significant differences observed for the model with the shorter MPA. The velocity streamlines depict recirculation zones for all four cases. Both models with parabolic inlet profiles and the model with the longer MPA and uniform profile exhibited similar recirculation zones, with a maximum difference of $2 \%$ for the longer MPAs. A smaller recirculation area was noticed for the shorter MPA model with uniform inlet profile, the length of which was about $10.5 \%$ smaller compared to that of the same-length MPA case with parabolic inlet profile (right column of Fig. 2a).

Fig. $2 \mathrm{~b}$ displays non-dimensionalised WSS profiles. No apparent differences were found between the WSS distributions of the models with different MPA lengths but the same parabolic inlet profile. A considerable decrease in the WSS was, nevertheless, observed along the inner wall of the model with uniform inlet and long MPA, which was further reduced for the model with the shorter MPA. To quantify the decrease, the integral of the WSS distribution was calculated for each case and compared with the integral of the parabolic inlet and long MPA model. A small decrease of $2 \%$ is found in the integral of the WSS of the model with the parabolic inlet profile and shorter MPA; the difference is increased to $\sim 27.7 \%$ and $53 \%$ for the models with the long and shorter MPA lengths with the uniform profile, respectively.
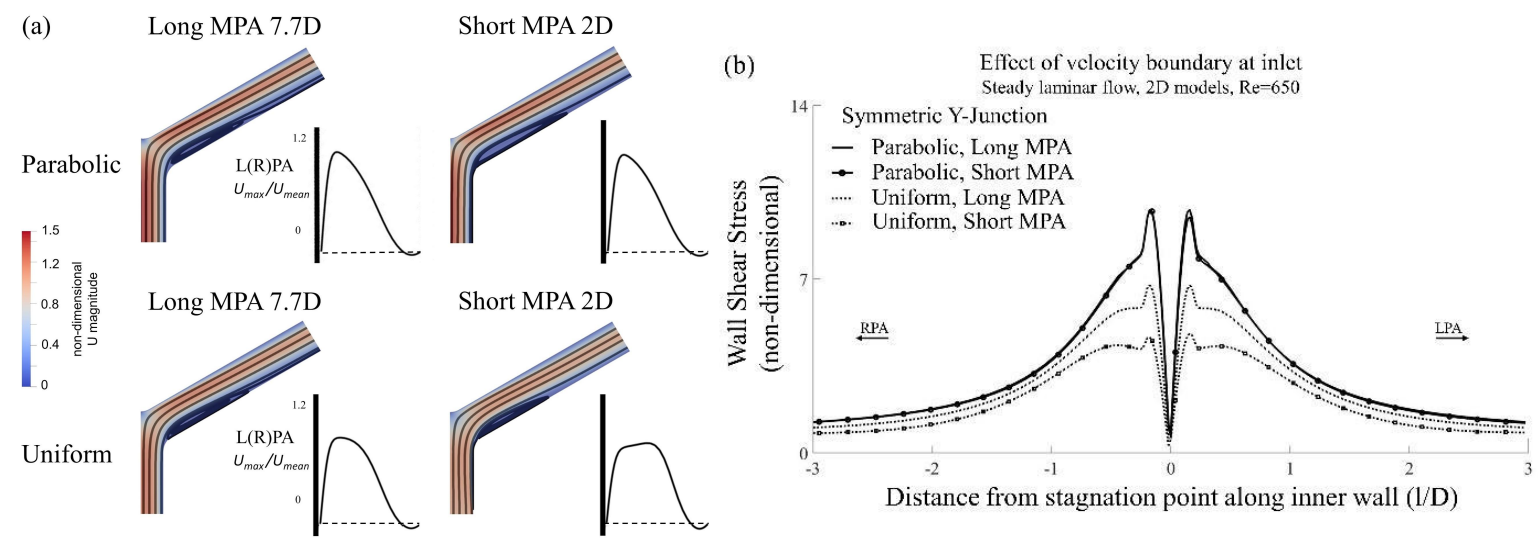

Fig. 2: Effect of steady inlet velocity profile with varied MPA lengths for the symmetric Y-Junction. (a) Non-dimensionalised velocity contours, overlaid by velocity streamlines, for parabolic and uniform inlet profiles (only half of the geometry is shown due to axisymmetry). Insets at the bottom right display velocity profiles within the RPA or LPA. (b) Non-dimensionalised WSS 
along the inner wall for parabolic and uniform inlet velocity profiles and different MPA lengths. Steady Newtonian flow, $R e=650$.

\section{Unsteady inlet flow}

For the time-dependent sinusoidal inlet profile of Eq. (1), a decrease of approximately $28 \%$ in the length of the recirculation zone was found based on the time-averaged velocity streamlines (Fig. 3a-ii), as compared with those for steady-state inlet flow (Fig. 3a-i). Only a single profile is presented in Fig. $3 a$ due to symmetry of the branches. Time-averaged wall shear stress for the unsteady case, nondimensionalised by the WSS value at the inlet wall, was further plotted along the inner wall of the arterial models (Fig. 4a). Although the WSS distribution was similar in shape for both steady and unsteady cases, the time-averaged WSS of the unsteady flow was slightly higher $(1.66 \%$ difference in the integral of the WSS). The influence of pulsatility in the WSS distribution is discussed in previous studies and similarities are reported between the steady state and time-averaged results [7-9]. A difference of up to $7 \%$ between time-averaged and steady wall shear is reported by Baidar and Cervantes (2017) [7] in simulations performed at a test case in a pipe and utilising different turbulent flow models, suggesting that steady simulations can capture time-averaged results. In a similar conclusion came Jodko et al (2016) [8], with simulation performed in patient-specific models of the fistula when they compared pulsating and stationary flow, and the two reached comparable results with the stationary simulations greatly reducing computational time. Kazakidi et al. (2011) [9], in an investigation performed in models of aortic branches to examine the effect of pulsatility found that the time averaged WSS was similar to the WSS of the steady flow case.

\section{Turbulent flow model}

Streamlines of velocity for the laminar (Fig. 3a-i) and the turbulent flow models (Fig. 3b-i) were compared for a Reynolds number of 650 and parabolic inlet velocity profile. A decrease in the recirculation area was noticed for the turbulent flow model, with the length of the recirculation zone reduced by approximately $23.3 \%$. The non-dimensionalised WSS profiles for the turbulent and laminar flow models, using parabolic inlet velocity profile, demonstrated a considerable decrease in the turbulent flow model (Fig. 4b). However, for a uniform inlet profile, the WSS profiles along the inner wall were almost identical for both the turbulent and laminar flow models (also in Fig. 4b) and very similar to the turbulent flow solution with parabolic velocity profile at the inlet. A possible explanation could be the dissipation in the velocity in the turbulent model, since the velocity is no longer parabolic and resembles more of a uniform profile. Therefore, flow enters the pulmonary junction with a lower maximum velocity compared to the equivalent value for the laminar model.

\section{Reynolds number with turbulent flow model}

An investigation on the effect of different Reynolds numbers on the velocity streamlines and WSS are shown in Fig. 3b and Fig. 4c, respectively. The length of the recirculation zone was increased at higher Reynolds numbers, by $14.2 \%$ and $17 \%$, for $\mathrm{Re}=1950$ and $\mathrm{Re}=3250$, respectively. Non-dimensionalised velocity profiles, based on division with the mean velocity at the entrance of the MPA for each Reynolds number respectively, were plotted at a distance of $3 \mathrm{~cm}(1.15 D)$ from the branch origin (Fig. 3b). The maximum forward velocities in the daughter branches slightly decreased in magnitude as the Reynolds number increased, with an equivalent increase in the retrograde flow, and the overall velocity profile was more uniform due to the higher inertia of the fluid particles at the middle of the branch at higher Reynolds numbers. The non-dimensionalised WSS profiles along the inner wall of the arterial models (Fig. 4c) were increased in magnitude with increasing Reynolds number. The effect of increasing Reynolds numbers on WSS values around arterial junctions (Fig. 4c) is in agreement with previous studies of similar effects [10]. Kazakidi et al. (2009) [10] investigated the effects of Reynolds number on patterns of WSS in idealised branching models of the intercoastal arteries and found that increasing the Re from 30 to 1500 led to enlarged regions of high WSS at the branch orifices.

\section{Flow in 3D model}

The steady and time-averaged velocity streamlines, as presented in Fig. 3c, indicate extended recirculation zones in both cases, with no major differences between the two, indicating the quasi-steady 
nature of the flow. In-plane velocity streamlines are also presented, taken at distance of $1.7 \mathrm{~cm}(\sim 0.65 \mathrm{D})$ from the stagnation point (arrows on the 3D geometry indicate the point of slice section) (Fig. 3c). Two counter rotating vortices were visible which is the result of secondary flow motion. Higher velocities from near-wall locations rotated first towards the inner wall and then the centre branch [11]. Nondimensionalised WSS profiles were further plotted (Fig. 4a) and were compared with WSS in the 2D models. WSS was significantly higher in the 3D models as compared to the $2 \mathrm{D}$ models, presenting up to $50 \%$ difference in the integrals of WSS for the steady flow and $52 \%$ difference in the unsteady cases. However, despite the quantitative difference, the WSS distribution was similar in shape for both 2D and 3D models, and steady or unsteady flow. The time-averaged WSS for the unsteady 3D flow was further elevated compared to the steady $3 \mathrm{D}$ flow, and a $2.84 \%$ difference in the integrals of the WSS was found between the two. The WSS distribution along the inner wall of the 3D models (Fig. 4a) is in close agreement with previous studies [12,13], even though the WSS values are somewhat lower in the 2D models (Fig. 4a).

(a) Effect of pulsatility

(i) Steady flow

Solution with laminar model, $\mathrm{Re}=650$

(b) Effect of turbulent flow model and Reynolds number Solution with turbulent model

(c) Effect of 3D model Solution with laminar model, $\mathrm{Re}=650$

(i) Steady flow

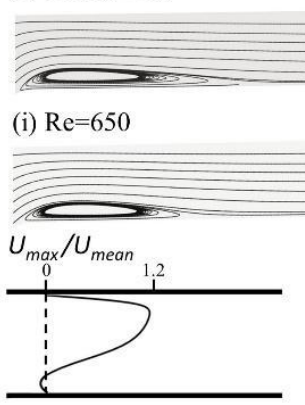

(ii) Unsteady flow

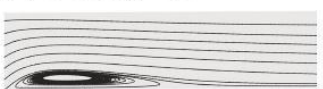

(iii) $\mathrm{Re}=3250$

(ii) $\mathrm{Re}=1950$
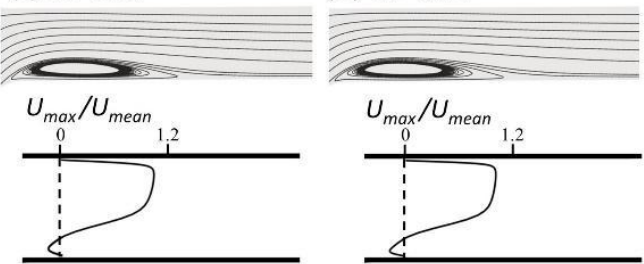

(ii) Unsteady flow
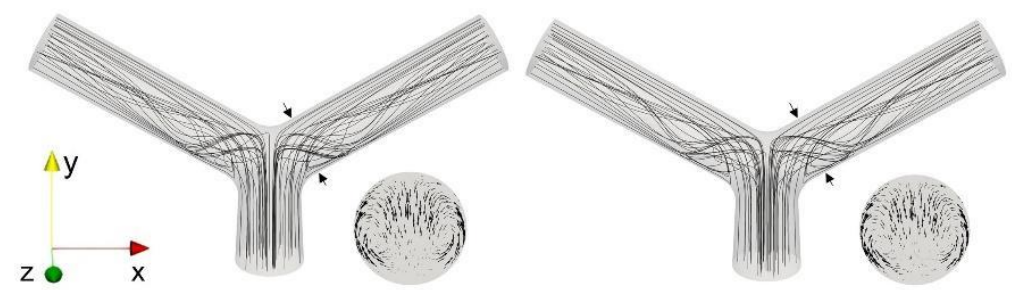

Fig. 3: Streamlines of velocity for different boundary conditions tested in the symmetric Y-Junction (Fig. 1f). Effects of: (a) pulsatile flow; (b) turbulent flow and Reynolds number (including velocity profiles, non-dimensionalised by division with the mean velocity at the inlet of MPA); and (c) three-dimensional flow. 

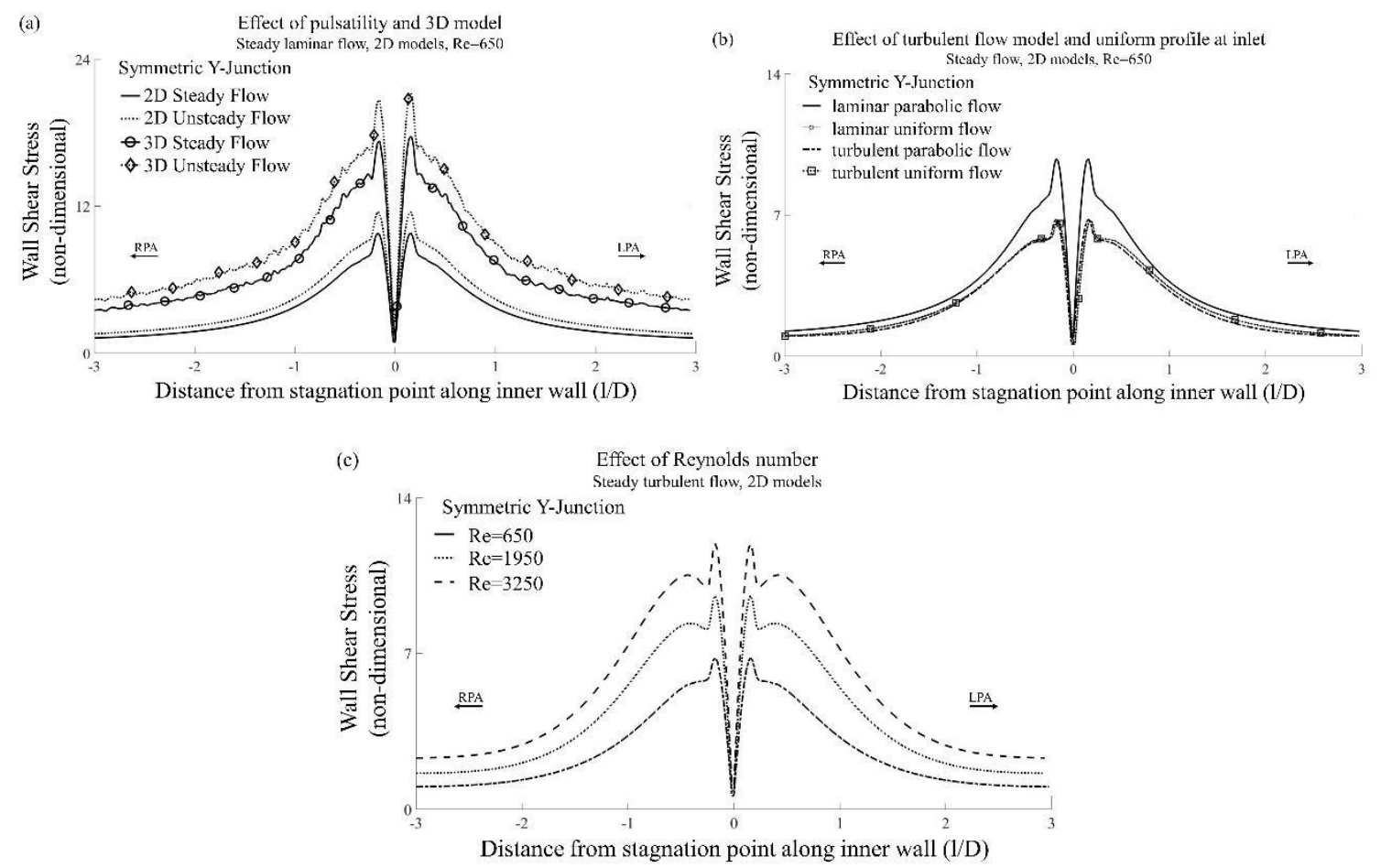

Fig. 4: Non-dimensional wall shear stress distribution, plotted along the inner wall of the arterial models for (a) steady and unsteady flow, in the $2 \mathrm{D}$ and $3 \mathrm{D}$ models; (b) laminar and turbulent models with parabolic and uniform inlet velocity $(\mathrm{Re}=650)$; (c) different Reynolds numbers assuming turbulence flow.

\section{Non-Newtonian models}

Finally, four different non-Newtonian models were tested: the power law, the Cross power law, the Casson, and the Bird-Carreau, and were compared with the Newtonian model. WSS was expressed in both non-dimensionalised form (Fig. 5a), based on the WSS value at the inlet MPA wall of each respective model (see Table 2), and in dynes $/ \mathrm{cm}^{2}$ (Fig. 5b).

In Fig. 5a, the WSS values proximal to the stagnation point varied considerably depending on the non-Newtonian model. The highest non-dimensionalised WSS values were found for the Newtonian model, while the lowest values were exhibited with the power law and Bird-Carreau models, which were very similar. Due to the non-dimensionalisation, all the WSS values along the inner wall distal to the stagnation point tended to unity, therefore there was not much variation in the WSS distal to the junction.

WSS values expressed in dynes $/ \mathrm{cm}^{2}$ (Fig. 5b) result in a slightly different distribution than in nondimensionalised form (Fig. 5a). The largest deviations in the WSS values existed at locations at a short distance from the stagnation point. The Newtonian model resulted in the highest WSS values, while the power law in the lowest values. Nevertheless, the Casson model displayed the highest similarity with the Newtonian model, while the power law appeared the least appropriate, showing the highest deviation from the Newtonian and the rest of the non-Newtonian models, in both Fig. 5a, 5b, proximal to the bifurcation. The WSS developed at the inner wall distal to the stagnation point vary slightly among the different models, with the largest distal deviation from the Newtonian model by the BirdCarreau model.

The WSS profiles calculated for the non-Newtonian models (Fig. 5) are, comparable with the results from the study of Karimi et al. (2014) [6]. In their work they study the blood flow in models of the human aorta and compare wall shear stress distribution in different rheological models. They observe similar wall shear stress patterns for all the models, but with variations in the magnitude of the wall shear stress. They conclude that the Cross model exhibited the highest discrepancies with the Newtonian and the other non-Newtonian models and recommend not to be used as an alternative rheological model. Finally, they observed an underestimation of the low WSS values with the Newtonian model [6]. In our 
study, the power law and the Cross power law showed the highest deviations from the rest of the models (Fig. 5b). The Newtonian model had lower wall shear stress values in the regions of low WSS but not deviating much from the Casson and Bird-Carreau models.
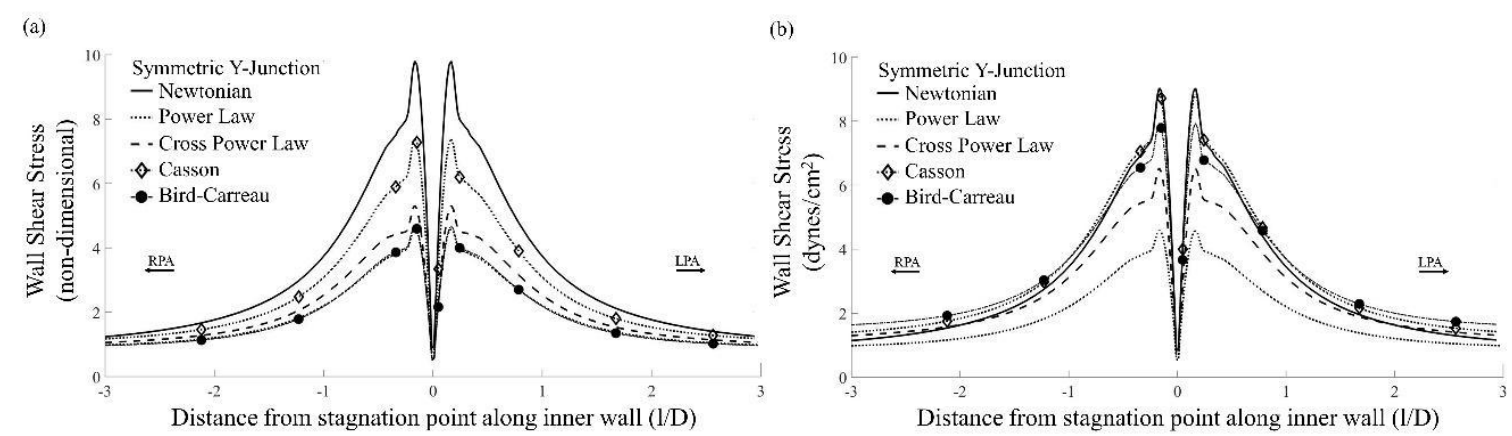

Fig. 5: WSS plots along the inner wall of the symmetric 2D Y-Junction (Fig. 1f) for the Newtonian and non-Newtonian models (a) non-dimensionalised form; (b) expressed in dynes $/ \mathrm{cm}^{2}$. Steady flow, $R e=650$.

\section{References}

[1] C. Caro, T. Pedley, R. Schroter, W. Seed, K. Parker, The mechanics of the Circulation. Cambridge: Cambridge University Press. (2011).

[2] M.U. Qureshi, N.A. Hill, A computational study of pressure wave reflections in the pulmonary arteries. Journal of Mathematical Biology (71) (2015) 1525-1549.

[3] A. Young, Patient-Specific Mathematical Modelling of the Hybrid Procedure in the Treatment of Hypoplastic Left Heart Syndrome, PhD thesis, University of Strathclyde, Glasgow (2014).

[4] F.R. Menter, Two-Equation Eddy-Viscosity Turbulence Models for Engineering Applications. AIAA Journal (32) (1994) 1598-1605.

[5] M.J. Chern, M.T. Wu, S. Wei-Her, Numerical Study for Blood Flow in Pulmonary Arteries after Repair of Tetralogy of Fallot. Computational and Mathematical Methods in Medicine (2012) (2012)118.

[6] S. Karimi, M. Dadagh, P. Vasava, M. Dadvar, B. Dadir, P. Jalali, Effect of rheological models on the hemodynamics within human aorta: CFD study on CT image-based geometry. Journal of NonNewtonian Fluid Mechanics (207) (2014) 42-52.

[7] B. Baidar, M.J. Cervantes, Numerical study of wall shear and velocities using a commercial CFD code: Some crucial aspects to consider. Hydro Nepal (21) (2017) 45-49.

[8] D. Jodko, D. Obidowski, P. Reorowicz, K. Jozwik, Numerical investigation of the unsteady blood flow in the end-to-side arteriovenous fistula for hemodialysis. Acta of Bioengineering and Biomechanics (18) (2016) 3-13.

[9] A. Kazakidi, A.M. Plata, S.J. Sherwin, P.D. Weinberg, Effect of reverse flow on the pattern of wall shear stress near arterial branches. Journal of the Royal Society Interface (8) (2011) 1594-1603.

[10] A. Kazakidi, S.J. Sherwin, P.D. Weinberg, Effect of Reynolds number and flow division on patterns of haemodynamic wall shear stress near branch points in the descending thoracic aorta. Journal of the Royal Society Interface (6) (2009) 539-548.

[11] T.J. Pedley, R.C. Schroter, M.F. Sudlow, Flow and pressure drop in systems of repeatedly branching tubes. Journal of Fluid Mechanics (46) (1971) 365-383.

[12] B.T. Tang, S.S. Pickard, F.P. Chan, P.S. Tsao, C.A. Taylor, J.A. Feinstein, Wall shear stress is decreased in the pulmonary arteries of patients with pulmonary arterial hypertension: An image-based, 
computational fluid dynamics study. Pulmonary Circulation, (2) (2012) 470-476.

[13] K. Kanokjaruvijit, T. Donprai-on, N. Phanthura, P. Noidet, J. Siripokharattana, Wall shear stress and velocity distributions in different types of stenotic bifurcations. Journal of Mechanical Science and Technology (31) (2017) 2339-2349. 


\section{Supplementary Methods}

A sensitivity analysis was performed in the symmetric Y-Junction model to investigate the effects of various different boundary conditions on the original solution, as follows:

Tapering in the bifurcation: Tapering is an unequivocal characteristic of arterial conduits, from the larger arteries to the smaller ones, based on multiple studies [1,2]. To investigate this effect a geometry was created, with the proximal main pulmonary artery (MPA), left pulmonary artery (LPA), and right pulmonary artery (RPA) RPA diameters of the tapered model kept equal to the non-tapered model, and the distal diameters modified to $\sim 0.95 D$ for the MPA and $\sim 0.75 \mathrm{D}$ for both daughter branches; $D$ is the diameter of the main pulmonary artery (MPA) assumed $2.6 \mathrm{~cm}$. These reductions in diameters correspond to a $4 \%$ and a $2 \%$ tapering, respectively, which are representative of the percentages found in a healthy young male [2]. No difference was found for the integral of the streamwise velocity profile between the non-tapered and tapered asymmetric models, as measured at the entrance of the bifurcation (at a distance $0.01 \mathrm{~m}$ from the origin $\mathrm{O}$ ).

An additional three-dimensional (3D) symmetric Y-Junction geometry was created based on the two-dimensional tapered model. The computational mesh of the 3D geometry consisted primarily of prismatic elements, with tetrahedral elements near the walls. A boundary layer was added near the arterial wall boundaries to capture the viscous effects, which included 17 layers with the first layer being at a distance of $\sim 0.002 D$ away from the wall. The total number of elements was $\sim 3800000$.

Velocity profile at the inlet and MPA length: The sensitivity of the steady velocity profile at the inlet of the MPA was investigated, first, through the use of a uniform inlet profile to represent blood flow emanating from the pulmonary valve, and, second, by reducing the length of the MPA to $\sim 2 D$ (equal to $5 \mathrm{~cm}$ ), in order to reflect a more physiological MPA dimension [2]. The uniform velocity profile was assumed with the same mean $U$ value as the parabolic profile.

Time-dependent flow: To account for the unsteady nature of blood flow, a sinusoidal variation of the velocity profile at the MPA inlet was assumed, based on the following expression:

$$
u(x, t)=u_{\text {par }}[1+\sin (2 \pi f t)]
$$

where $u_{\text {par }}(x)$ is given by Eq. (2),

$$
u_{p a r}(x)=\frac{3}{2} U\left(1-\frac{4 x^{2}}{D^{2}}\right)
$$

and $f$ is the frequency, assumed as $2 \mathrm{~Hz}$ [3]. Time-averaged results over a single cycle were compared with the steady-state numerical results for the same mean velocity.

Turbulent flow model: The simulations were performed assuming the k- $\omega$ shear stress transport (SST) turbulence flow model (with model specifications as presented in Table 1) [4], calculated using the following formulas: turbulent intensity $\left(I=0.16^{*} R e_{D}{ }^{-1 / 8}\right)$, turbulent energy $\left(k=3 / 2 *(U I)^{2}\right)$, turbulent length scale $\left(L=0.038^{*} D\right)$, and specific dissipation rate $(\omega=\sqrt{k} / L)$.

Reynolds number with turbulent flow model: The effect of Reynolds number was tested by increasing the Reynolds number from 650 to 1950 and 3250, which correspond to an increase of the mean velocity $U$ from $0.1 \mathrm{~m} / \mathrm{s}$ to $0.3 \mathrm{~m} / \mathrm{s}$ and $0.5 \mathrm{~m} / \mathrm{s}$, respectively, for the specific MPA diameter, D. Such values of velocities can be observed in the pulmonary bifurcation during systole [5]. The simulations were performed using the turbulent flow model.

Table 1: Parameters used for the k- $\omega$ SST turbulent model (U: mean velocity, Re: Reynolds number, I: turbulence intensity,

\begin{tabular}{|c|c|c|c|c|c|}
\hline$U(\mathrm{~m} / \mathrm{s})$ & $\mathbf{R e}$ & I & $k\left(m^{2} / s^{2}\right)$ & $L(\mathrm{~m})$ & $\Omega(1 / s)$ \\
\hline 0.1 & 650 & 0.071 & $7.6 e-05$ & $9.88 \mathrm{e}-04$ & 8.83 \\
\hline 0.3 & 1950 & 0.062 & $5.2 \mathrm{e}-4$ & $9.88 \mathrm{e}-04$ & 23.08 \\
\hline 0.5 & 3250 & 0.058 & $1.2 \mathrm{e}-03$ & $9.88 \mathrm{e}-04$ & 36.10 \\
\hline
\end{tabular}
$k$ : turbulent energy, $l:$ turbulent length scale, and $\omega$ : specific dissipation rate). 
Inlet flow condition for the three-dimensional model: The three-dimensional symmetric Y-Junction was tested by assuming a fully developed velocity profile at the MPA inlet, for both steady and unsteady flow, with the same mean velocity $U$.

Non-Newtonian models: Additional simulations considered the non-Newtonian rheology of blood. For that, four different models were tested, namely the power law, the Cross power law, and the Casson and Bird-Carreau models, with model specifications presented in Table 2 [6]. The power law is the simplest non-Newtonian model to be considered. The Bird-Carreau and the Cross power law models have viscosities very close to the Newtonian one at high shear rates. In addition, the cross model considers a short rate of viscosities and therefore could be a good candidate for simulating blood flow in large arteries. Finally, the Casson model is expressed in terms of the hematocrit, which is assumed $40 \%$ for normal blood [6].

Table 2: Non-Newtonian models of blood flow ( $v$ : blood viscosity, $v_{\infty}$ : infinite shear rate viscosity, $v_{o}$ : zero shear rate viscosity, $k$ : relaxation time constant, $\dot{\gamma}$ : shear rate, $\alpha$ : constant parameter, $n:$ power law index, $\tau_{0}:$ yield stress). Wall shear stress values at the inlet MPA walls of the symmetric Y-Junction for the different models, are also presented.

\begin{tabular}{|c|c|c|c|}
\hline Rheological Model & Viscosity & Specified Values & WSS at inlet MPA wall (dynes $\left./ \mathrm{cm}^{2}\right)$ \\
\hline Newtonian & $v$ & $v: 0.004 \mathrm{~Pa} \mathrm{~s}$ & 0.922 \\
\hline Power Law & $v=(k \dot{\gamma})^{n-1}, v_{\min } \leq v \leq v_{\max }$ & $\begin{array}{l}\nu_{\max }: 0.056 \mathrm{~Pa} \mathrm{~s} \\
v_{\min }: 0.0035 \mathrm{~Pa} \mathrm{~s} \\
\mathrm{k}: 0.017 \mathrm{~s}, \mathrm{n}: 0.708\end{array}$ & 1.696 \\
\hline Cross power law & $v=v_{\infty}+\frac{v_{0}-v_{\infty}}{1+(m \dot{\gamma})^{\alpha}}$ & $\begin{array}{l}\nu_{\infty}: 0.0035 \mathrm{~Pa} \mathrm{~s}, \\
\nu_{o}: 0.0364 \mathrm{~Pa} \mathrm{~s}, \\
\mathrm{~m}: 0.38 \mathrm{~s} \text { (consistency } \\
\text { index), } \alpha: 1.45\end{array}$ & 1.229 \\
\hline Casson & $v=\left(\sqrt{\tau_{0} / \dot{\gamma}}+\sqrt{m}\right)^{2}$ & $\begin{array}{l}\mathrm{m}: 0.00414 \mathrm{~Pa} \mathrm{~s} \\
\text { (viscosity coefficient), } \\
\tau_{0}: 0.0038 \mathrm{~Pa}\end{array}$ & 1.198 \\
\hline Bird-Carreau model & $\begin{array}{l}v \\
=v_{\infty} \\
+\left(v_{o}-v_{\infty}\right)\left[1+(k \dot{\gamma})^{\alpha}\right]^{(n-1) / \alpha}\end{array}$ & $\begin{array}{l}v_{\infty}: 0.0035 \mathrm{~Pa} \mathrm{~s} \\
v_{0}: 0.056 \mathrm{~Pa} \mathrm{~s} \\
\mathrm{k}: 3.313 \mathrm{~s}, \mathrm{n}: 0.3568\end{array}$ & 1.696 \\
\hline
\end{tabular}

\section{Post-processing}

For the non-Newtonian model, WSS values were non-dimensionalised with values calculated at the inlet of a straight geometry with the same MPA diameter and flow conditions (last column of Table 2). The time-averaged wall shear stress was further used and was determined according to the following equation:

$$
\tau_{\text {mean }}=\frac{1}{T} \int_{0}^{T}\left|\tau_{w}\right| d t
$$

where $\left|\tau_{w}\right|$ is the magnitude of the instantaneous WSS vector.

\section{Verification and Validation}

The comparison between OpenFOAM ${ }^{\circledR}$ and ANSYS Fluent ${ }^{\circledR}$, mentioned in Section 2.7 of the manuscript, is shown in Fig. 1a for non-dimensionalised velocity contours. A percentage difference of $0.047 \%$ was quantified for the integral of velocity profiles (Fig. 1b), positioned at the entrance of the bifurcation (at a distance $0.01 \mathrm{~m}$ from the origin $\mathrm{O}$ ). 
(a)

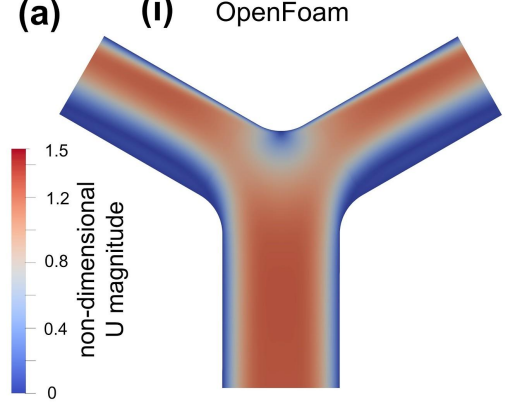

(ii)

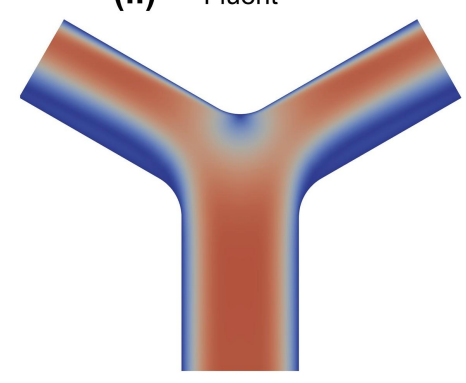

(b)

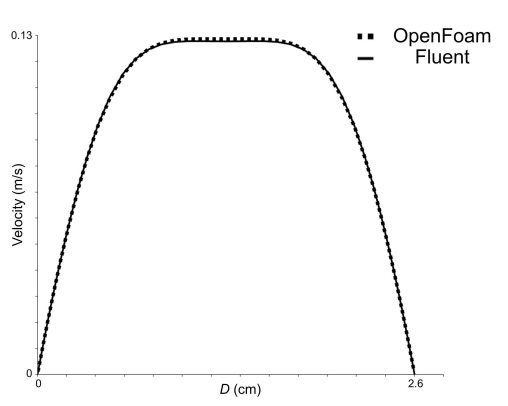

Fig. 1: Comparison of OpenFoam and Ansys Fluent. (a) Non-dimensionalised velocity contours. (b) Velocity profiles at the entrance of the bifurcation and at a distance of $0.01 \mathrm{~m}$ from the branch origin. Steady Newtonian flow, $R e=650$.

\section{Supplementary Results}

\section{Steady inlet velocity profile and MPA length}

Fig. 2a presents velocity contours overlaid by streamlines of velocity, and the insets show the respective velocity profiles, non-dimensionalised by the mean MPA velocity. The length of the MPA had no effect on the velocity distribution in the models with the same parabolic inlet profile, as expected (top row of Fig. 2a). However, there was an overall decrease in the maximum velocity values in the daughter branches, for the models with uniform inlet profiles, for both MPA lengths (bottom row of Fig. 2a), with the most significant differences observed for the model with the shorter MPA. The velocity streamlines depict recirculation zones for all four cases. Both models with parabolic inlet profiles and the model with the longer MPA and uniform profile exhibited similar recirculation zones, with a maximum difference of $2 \%$ for the longer MPAs. A smaller recirculation area was noticed for the shorter MPA model with uniform inlet profile, the length of which was about $10.5 \%$ smaller compared to that of the same-length MPA case with parabolic inlet profile (right column of Fig. 2a).

Fig. $2 \mathrm{~b}$ displays non-dimensionalised WSS profiles. No apparent differences were found between the WSS distributions of the models with different MPA lengths but the same parabolic inlet profile. A considerable decrease in the WSS was, nevertheless, observed along the inner wall of the model with uniform inlet and long MPA, which was further reduced for the model with the shorter MPA. To quantify the decrease, the integral of the WSS distribution was calculated for each case and compared with the integral of the parabolic inlet and long MPA model. A small decrease of $2 \%$ is found in the integral of the WSS of the model with the parabolic inlet profile and shorter MPA; the difference is increased to $\sim 27.7 \%$ and $53 \%$ for the models with the long and shorter MPA lengths with the uniform profile, respectively.
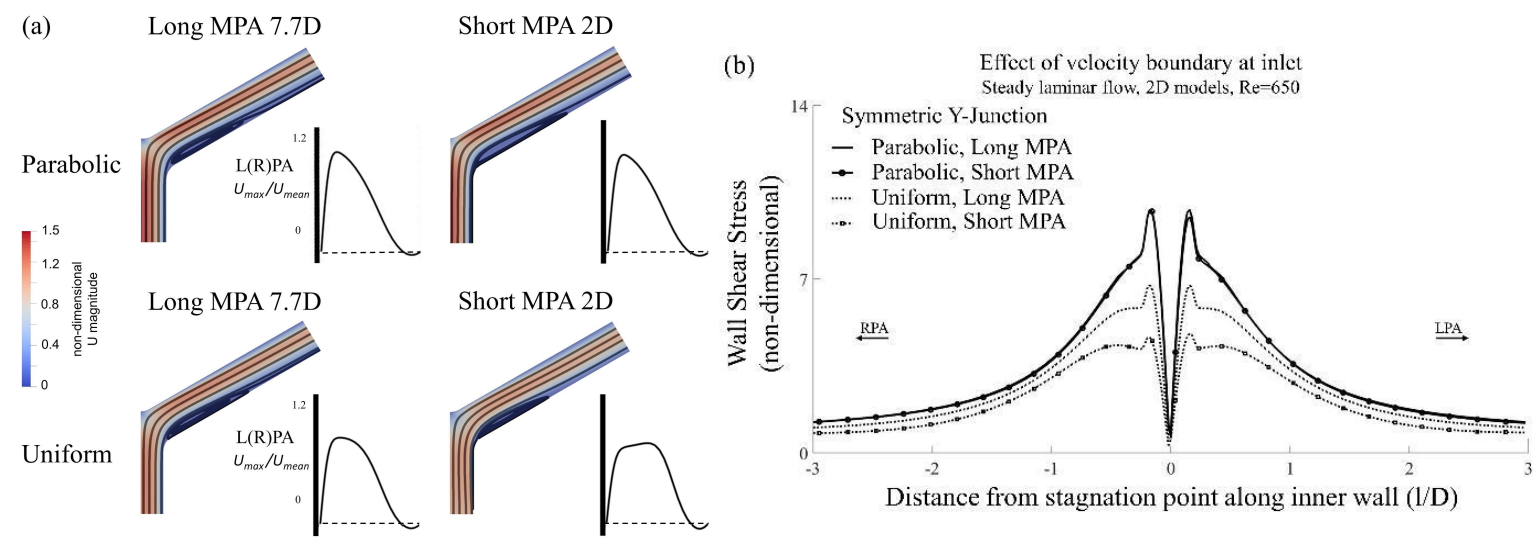

Fig. 2: Effect of steady inlet velocity profile with varied MPA lengths for the symmetric Y-Junction. (a) Non-dimensionalised velocity contours, overlaid by velocity streamlines, for parabolic and uniform inlet profiles (only half of the geometry is shown due to axisymmetry). Insets at the bottom right display velocity profiles within the RPA or LPA. (b) Non-dimensionalised WSS 
along the inner wall for parabolic and uniform inlet velocity profiles and different MPA lengths. Steady Newtonian flow, $R e=650$.

\section{Unsteady inlet flow}

For the time-dependent sinusoidal inlet profile of Eq. (1), a decrease of approximately $28 \%$ in the length of the recirculation zone was found based on the time-averaged velocity streamlines (Fig. 3a-ii), as compared with those for steady-state inlet flow (Fig. 3a-i). Only a single profile is presented in Fig. $3 a$ due to symmetry of the branches. Time-averaged wall shear stress for the unsteady case, nondimensionalised by the WSS value at the inlet wall, was further plotted along the inner wall of the arterial models (Fig. 4a). Although the WSS distribution was similar in shape for both steady and unsteady cases, the time-averaged WSS of the unsteady flow was slightly higher $(1.66 \%$ difference in the integral of the WSS). The influence of pulsatility in the WSS distribution is discussed in previous studies and similarities are reported between the steady state and time-averaged results [7-9]. A difference of up to $7 \%$ between time-averaged and steady wall shear is reported by Baidar and Cervantes (2017) [7] in simulations performed at a test case in a pipe and utilising different turbulent flow models, suggesting that steady simulations can capture time-averaged results. In a similar conclusion came Jodko et al (2016) [8], with simulation performed in patient-specific models of the fistula when they compared pulsating and stationary flow, and the two reached comparable results with the stationary simulations greatly reducing computational time. Kazakidi et al. (2011) [9], in an investigation performed in models of aortic branches to examine the effect of pulsatility found that the time averaged WSS was similar to the WSS of the steady flow case.

\section{Turbulent flow model}

Streamlines of velocity for the laminar (Fig. 3a-i) and the turbulent flow models (Fig. 3b-i) were compared for a Reynolds number of 650 and parabolic inlet velocity profile. A decrease in the recirculation area was noticed for the turbulent flow model, with the length of the recirculation zone reduced by approximately $23.3 \%$. The non-dimensionalised WSS profiles for the turbulent and laminar flow models, using parabolic inlet velocity profile, demonstrated a considerable decrease in the turbulent flow model (Fig. 4b). However, for a uniform inlet profile, the WSS profiles along the inner wall were almost identical for both the turbulent and laminar flow models (also in Fig. 4b) and very similar to the turbulent flow solution with parabolic velocity profile at the inlet. A possible explanation could be the dissipation in the velocity in the turbulent model, since the velocity is no longer parabolic and resembles more of a uniform profile. Therefore, flow enters the pulmonary junction with a lower maximum velocity compared to the equivalent value for the laminar model.

\section{Reynolds number with turbulent flow model}

An investigation on the effect of different Reynolds numbers on the velocity streamlines and WSS are shown in Fig. 3b and Fig. 4c, respectively. The length of the recirculation zone was increased at higher Reynolds numbers, by $14.2 \%$ and $17 \%$, for $\mathrm{Re}=1950$ and $\mathrm{Re}=3250$, respectively. Non-dimensionalised velocity profiles, based on division with the mean velocity at the entrance of the MPA for each Reynolds number respectively, were plotted at a distance of $3 \mathrm{~cm}(1.15 D)$ from the branch origin (Fig. 3b). The maximum forward velocities in the daughter branches slightly decreased in magnitude as the Reynolds number increased, with an equivalent increase in the retrograde flow, and the overall velocity profile was more uniform due to the higher inertia of the fluid particles at the middle of the branch at higher Reynolds numbers. The non-dimensionalised WSS profiles along the inner wall of the arterial models (Fig. 4c) were increased in magnitude with increasing Reynolds number. The effect of increasing Reynolds numbers on WSS values around arterial junctions (Fig. 4c) is in agreement with previous studies of similar effects [10]. Kazakidi et al. (2009) [10] investigated the effects of Reynolds number on patterns of WSS in idealised branching models of the intercoastal arteries and found that increasing the Re from 30 to 1500 led to enlarged regions of high WSS at the branch orifices.

\section{Flow in 3D model}

The steady and time-averaged velocity streamlines, as presented in Fig. 3c, indicate extended recirculation zones in both cases, with no major differences between the two, indicating the quasi-steady 
nature of the flow. In-plane velocity streamlines are also presented, taken at distance of $1.7 \mathrm{~cm}(\sim 0.65 \mathrm{D})$ from the stagnation point (arrows on the 3D geometry indicate the point of slice section) (Fig. 3c). Two counter rotating vortices were visible which is the result of secondary flow motion. Higher velocities from near-wall locations rotated first towards the inner wall and then the centre branch [11]. Nondimensionalised WSS profiles were further plotted (Fig. 4a) and were compared with WSS in the 2D models. WSS was significantly higher in the 3D models as compared to the 2D models, presenting up to $50 \%$ difference in the integrals of WSS for the steady flow and $52 \%$ difference in the unsteady cases. However, despite the quantitative difference, the WSS distribution was similar in shape for both 2D and 3D models, and steady or unsteady flow. The time-averaged WSS for the unsteady 3D flow was further elevated compared to the steady $3 \mathrm{D}$ flow, and a $2.84 \%$ difference in the integrals of the WSS was found between the two. The WSS distribution along the inner wall of the 3D models (Fig. 4a) is in close agreement with previous studies [12,13], even though the WSS values are somewhat lower in the 2D models (Fig. 4a).

(a) Effect of pulsatility

(i) Steady flow

Solution with laminar model, $\mathrm{Re}=650$

(b) Effect of turbulent flow model and Reynolds number Solution with turbulent model

(c) Effect of 3D model Solution with laminar model, $\mathrm{Re}=650$

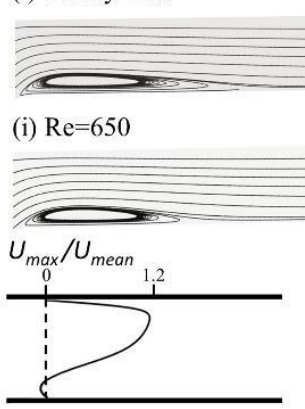

(i) Steady flow (ii) Unsteady flow

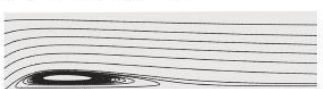

(iii) $\mathrm{Re}=3250$

(ii) $\mathrm{Re}=1950$
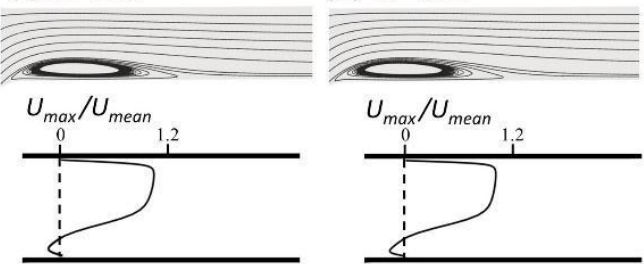

(ii) Unsteady flow
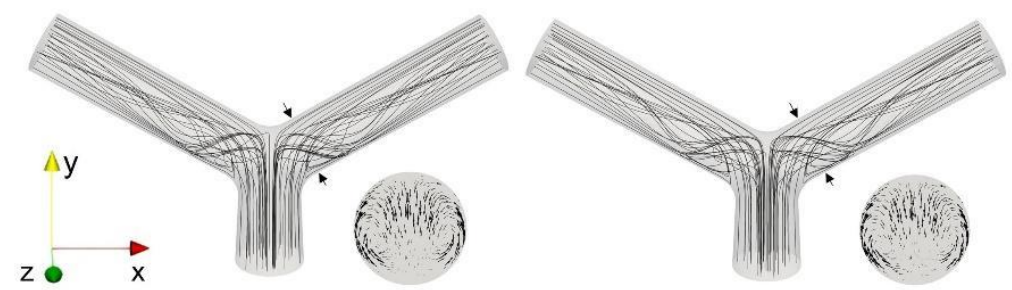

Fig. 3: Streamlines of velocity for different boundary conditions tested in the symmetric Y-Junction (Fig. 1f). Effects of: (a) pulsatile flow; (b) turbulent flow and Reynolds number (including velocity profiles, non-dimensionalised by division with the mean velocity at the inlet of MPA); and (c) three-dimensional flow. 

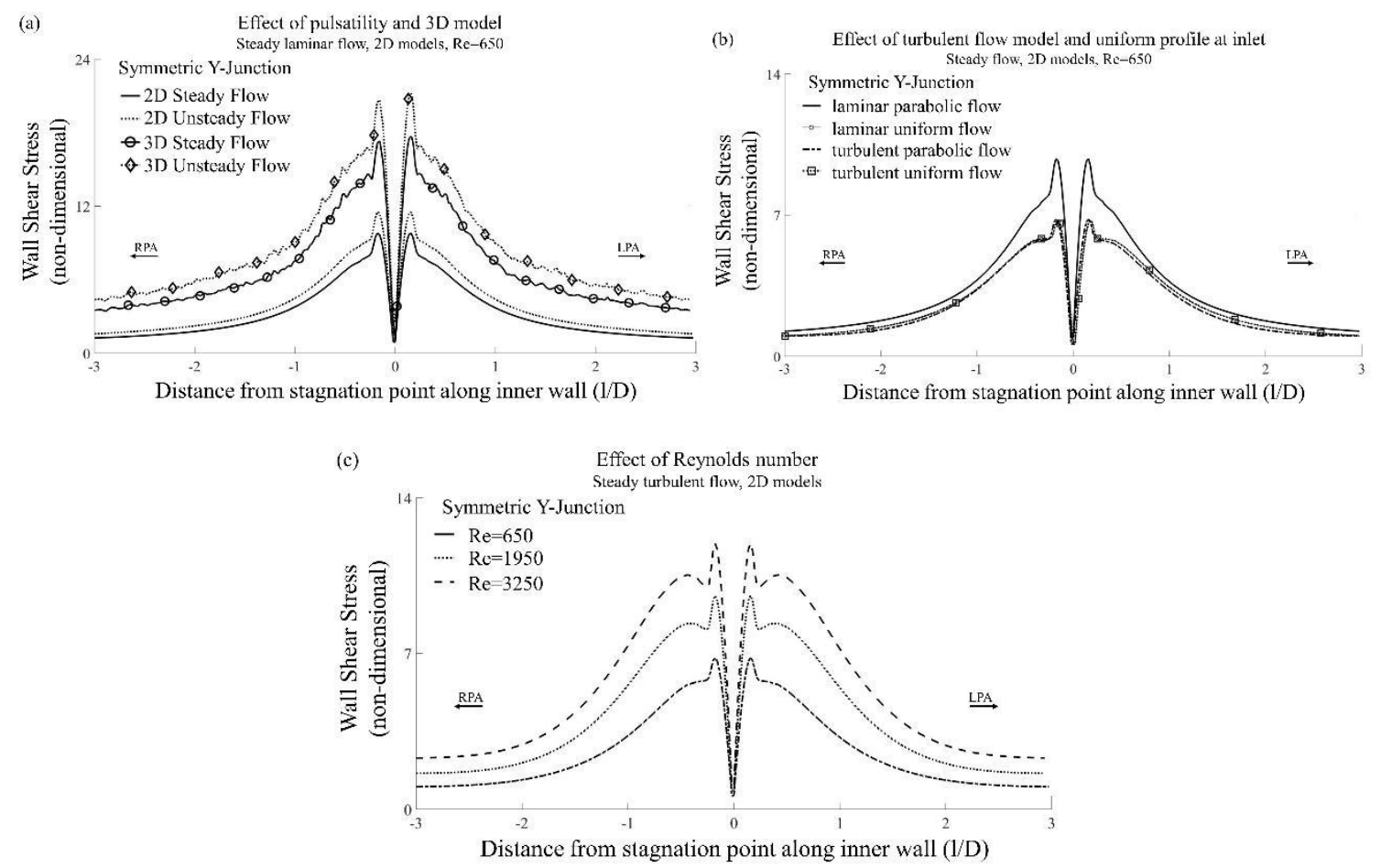

Fig. 4: Non-dimensional wall shear stress distribution, plotted along the inner wall of the arterial models for (a) steady and unsteady flow, in the $2 \mathrm{D}$ and $3 \mathrm{D}$ models; (b) laminar and turbulent models with parabolic and uniform inlet velocity $(\mathrm{Re}=650)$; (c) different Reynolds numbers assuming turbulence flow.

\section{Non-Newtonian models}

Finally, four different non-Newtonian models were tested: the power law, the Cross power law, the Casson, and the Bird-Carreau, and were compared with the Newtonian model. WSS was expressed in both non-dimensionalised form (Fig. 5a), based on the WSS value at the inlet MPA wall of each respective model (see Table 2), and in dynes $/ \mathrm{cm}^{2}$ (Fig. 5b).

In Fig. 5a, the WSS values proximal to the stagnation point varied considerably depending on the non-Newtonian model. The highest non-dimensionalised WSS values were found for the Newtonian model, while the lowest values were exhibited with the power law and Bird-Carreau models, which were very similar. Due to the non-dimensionalisation, all the WSS values along the inner wall distal to the stagnation point tended to unity, therefore there was not much variation in the WSS distal to the junction.

WSS values expressed in dynes $/ \mathrm{cm}^{2}$ (Fig. 5b) result in a slightly different distribution than in nondimensionalised form (Fig. 5a). The largest deviations in the WSS values existed at locations at a short distance from the stagnation point. The Newtonian model resulted in the highest WSS values, while the power law in the lowest values. Nevertheless, the Casson model displayed the highest similarity with the Newtonian model, while the power law appeared the least appropriate, showing the highest deviation from the Newtonian and the rest of the non-Newtonian models, in both Fig. 5a, 5b, proximal to the bifurcation. The WSS developed at the inner wall distal to the stagnation point vary slightly among the different models, with the largest distal deviation from the Newtonian model by the BirdCarreau model.

The WSS profiles calculated for the non-Newtonian models (Fig. 5) are, comparable with the results from the study of Karimi et al. (2014) [6]. In their work they study the blood flow in models of the human aorta and compare wall shear stress distribution in different rheological models. They observe similar wall shear stress patterns for all the models, but with variations in the magnitude of the wall shear stress. They conclude that the Cross model exhibited the highest discrepancies with the Newtonian and the other non-Newtonian models and recommend not to be used as an alternative rheological model. Finally, they observed an underestimation of the low WSS values with the Newtonian model [6]. In our 
study, the power law and the Cross power law showed the highest deviations from the rest of the models (Fig. 5b). The Newtonian model had lower wall shear stress values in the regions of low WSS but not deviating much from the Casson and Bird-Carreau models.
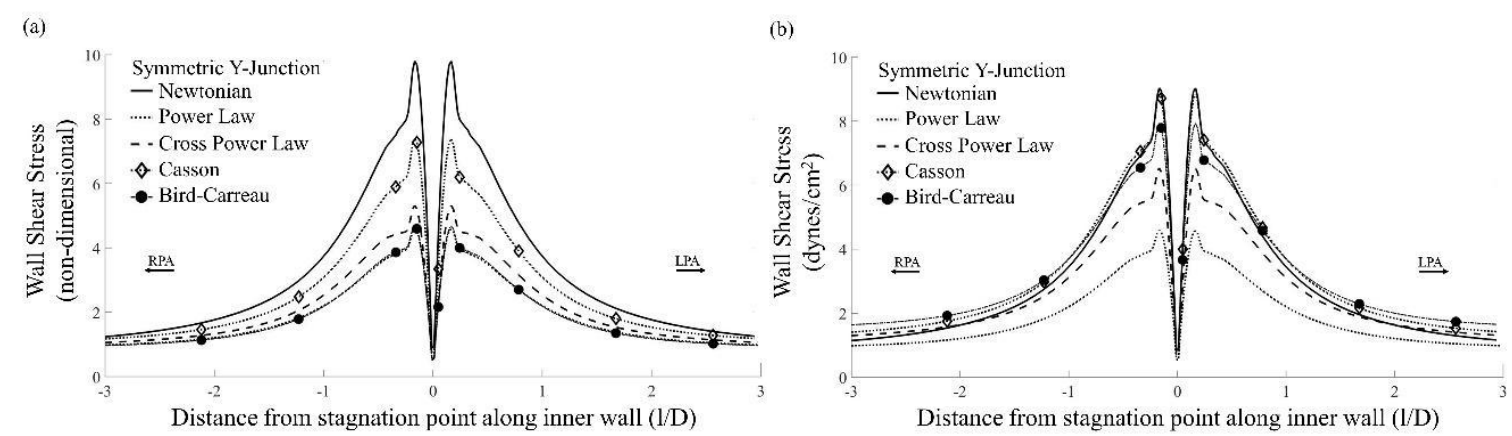

Fig. 5: WSS plots along the inner wall of the symmetric 2D Y-Junction (Fig. 1f) for the Newtonian and non-Newtonian models (a) non-dimensionalised form; (b) expressed in dynes $/ \mathrm{cm}^{2}$. Steady flow, $R e=650$.

\section{References}

[1] C. Caro, T. Pedley, R. Schroter, W. Seed, K. Parker, The mechanics of the Circulation. Cambridge: Cambridge University Press. (2011).

[2] M.U. Qureshi, N.A. Hill, A computational study of pressure wave reflections in the pulmonary arteries. Journal of Mathematical Biology (71) (2015) 1525-1549.

[3] A. Young, Patient-Specific Mathematical Modelling of the Hybrid Procedure in the Treatment of Hypoplastic Left Heart Syndrome, PhD thesis, University of Strathclyde, Glasgow (2014).

[4] F.R. Menter, Two-Equation Eddy-Viscosity Turbulence Models for Engineering Applications. AIAA Journal (32) (1994) 1598-1605.

[5] M.J. Chern, M.T. Wu, S. Wei-Her, Numerical Study for Blood Flow in Pulmonary Arteries after Repair of Tetralogy of Fallot. Computational and Mathematical Methods in Medicine (2012) (2012)118.

[6] S. Karimi, M. Dadagh, P. Vasava, M. Dadvar, B. Dadir, P. Jalali, Effect of rheological models on the hemodynamics within human aorta: CFD study on CT image-based geometry. Journal of NonNewtonian Fluid Mechanics (207) (2014) 42-52.

[7] B. Baidar, M.J. Cervantes, Numerical study of wall shear and velocities using a commercial CFD code: Some crucial aspects to consider. Hydro Nepal (21) (2017) 45-49.

[8] D. Jodko, D. Obidowski, P. Reorowicz, K. Jozwik, Numerical investigation of the unsteady blood flow in the end-to-side arteriovenous fistula for hemodialysis. Acta of Bioengineering and Biomechanics (18) (2016) 3-13.

[9] A. Kazakidi, A.M. Plata, S.J. Sherwin, P.D. Weinberg, Effect of reverse flow on the pattern of wall shear stress near arterial branches. Journal of the Royal Society Interface (8) (2011) 1594-1603.

[10] A. Kazakidi, S.J. Sherwin, P.D. Weinberg, Effect of Reynolds number and flow division on patterns of haemodynamic wall shear stress near branch points in the descending thoracic aorta. Journal of the Royal Society Interface (6) (2009) 539-548.

[11] T.J. Pedley, R.C. Schroter, M.F. Sudlow, Flow and pressure drop in systems of repeatedly branching tubes. Journal of Fluid Mechanics (46) (1971) 365-383.

[12] B.T. Tang, S.S. Pickard, F.P. Chan, P.S. Tsao, C.A. Taylor, J.A. Feinstein, Wall shear stress is decreased in the pulmonary arteries of patients with pulmonary arterial hypertension: An image-based, 
computational fluid dynamics study. Pulmonary Circulation, (2) (2012) 470-476.

[13] K. Kanokjaruvijit, T. Donprai-on, N. Phanthura, P. Noidet, J. Siripokharattana, Wall shear stress and velocity distributions in different types of stenotic bifurcations. Journal of Mechanical Science and Technology (31) (2017) 2339-2349. 